\title{
Scaling patterns for QCD jets
}

\section{Erik Gerwick, ${ }^{a}$ Tilman Plehn, $^{b}$ Steffen Schumann ${ }^{a}$ and Peter Schichtel ${ }^{b}$}

\author{
${ }^{a}$ II. Physikalisches Institut, Universität Göttingen, \\ Göttingen, Germany \\ ${ }^{b}$ Institut für Theoretische Physik, Universität Heidelberg, \\ Heidelberg, Germany \\ E-mail: erik.gerwick@phys.uni-goettingen.de, plehn@uni-heidelberg.de, \\ steffen.schumann@phys.uni-goettingen.de, \\ p.schichtel@thphys.uni-heidelberg.de
}

\begin{abstract}
Jet emission at hadron colliders follows simple scaling patterns. Based on perturbative QCD we derive Poisson and staircase scaling for final state as well as initial state radiation. Parton density effects enhance staircase scaling at low multiplicities. We propose experimental tests of our theoretical findings in $\mathrm{Z}+$ jets and QCD gap jets production, based on minor additions to current LHC analyses.
\end{abstract}

KeYwords: Jets, Monte Carlo Simulations

ARXIV EPRINT: 1208.3676 


\section{Contents}

1 Multi-jet rates 1

2 Final state splittings $\quad 4$

2.1 Final-state parton shower 4

$\begin{array}{lll}2.2 & \text { Generating functional for jet fractions } & 7\end{array}$

2.3 Matrix element corrections 11

3 Hadron colliders $\quad 12$

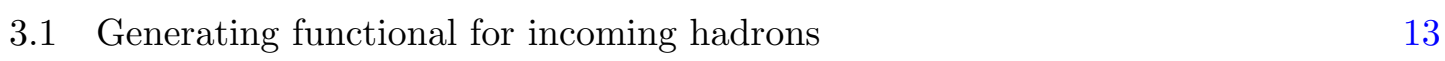

$\begin{array}{lll}3.2 & \text { Parton density suppression } & 16\end{array}$

$\begin{array}{lll}3.3 & \text { Initial-state parton shower } & 17\end{array}$

$\begin{array}{llr}3.4 & \text { BFKL evolution } & 19\end{array}$

4 LHC predictions $\quad 20$

$\begin{array}{lll}4.1 & \mathrm{Z}+\text { jets } & 21\end{array}$

4.2 QCD gap jets 23

5 Conclusions $\quad 25$

$\begin{array}{lr}\text { A Iterated Poisson process } & 26\end{array}$

$\begin{array}{ll}\text { B Jet fractions } & 29\end{array}$

\section{Multi-jet rates}

Multi-jets final states are ubiquitous at hadron colliders. QCD jet radiation mostly off the initial state partons has huge impact on almost every LHC analysis. For example in Higgs searches an accurate description of the jet recoil against the Higgs boson allows for an efficient rejection of many backgrounds [1]. In top pair production and single top production the identification of QCD recoil jets on the one hand and decay jets on the other hand is one of the limiting factors in precision analyses [2-4]. New physics searches largely rely on hard decay jets of new strongly interacting particles, which makes them vulnerable in the case of soft decay jets hidden in the QCD jet activity [5-9]. Understanding the jet multiplicity and the jet spectra from QCD radiation is a core ingredient to improving any of these analyses.

Even though jet radiation seems to follow simple patterns [10-13], theoretical predictions for multi-jet observables in perturbative QCD are challenging. It is clear that the radiation of relatively hard quarks and gluons is a direct consequence of the hierarchy between the large proton-proton collider energy and the typical electroweak scale of the 
partonic interaction [14, 15]. Numerically, we can combine the QCD parton shower with hard matrix element calculations, to predict jet radiation patterns over wide phase space regions ([16-18]; for a pedagogical introduction see e.g. [19]). Analytically, Sudakov factors and generating functionals can be used to describe QCD jet radiation [20,21]. A careful comparison of analytical and numerical approaches to LHC data would allow us to determine the strengths and limitations of the underlying theoretical concepts: fixed-order perturbation theory, parton showers, and resummation based on generating functionals.

In fixed order perturbation theory leading-order jet rates are available for effectively arbitrarily high multiplicities $[22,23]$. The number of jets we can consider is limited only by computing power. However, leading order predictions suffer significant shortcomings when it comes to precision. The renormalization scale dependence as a measure for the theoretical uncertainty grows with each power of $\alpha_{s}$ when we add a final state jet. High powers of the logarithmic scale dependence $\left(\log \mu_{R}\right)^{n}$ mean that such cross section predictions can only be considered an order-of-magnitude estimate. In addition, in the presence of phase space constraints large logarithms spoil the convergence of fixed order perturbation theory. For example jet vetoes will induce sizeable logarithms of ratios of leading jet $p_{T}$ or masses $\left(m_{H}\right.$ or $m_{Z}$ ) to additional resolved jets (experimentally relevant down to $20-30 \mathrm{GeV}$ ) [24, 25].

Next-to-leading order computations ameliorate the scale dependence and capture an additional logarithm. However, we are still limited in the available final-state multiplicity, e.g. pure jets are available for $n_{\text {jets }} \leq 4$ [26], $n_{\text {jets }} \leq 4$ in association with $W / Z$ bosons [27, $28]$, for $t \bar{t}$ production and Higgs production in gluon fusion NLO corrections are known for $n_{\text {jets }} \leq 2$ [29-33]. However, over the past few years this field has progressed enormously. As a consequence for Standard Model processes a similar level of automation as for leadingorder calculations is within reach [34-43]. An approach applicable for general New Physics extensions, though limited to $2 \rightarrow 2$ processes, has been presented in [44, 45].

Although NLO calculations contain one additional power of enhanced logarithms, this might not be sufficient for high jet multiplicities. At NNLO, although there has been an enormous amount of recent development [46-48], the number of fully differential calculations is limited, and an automated implementation is not foreseeable in the near future.

On the other hand, we know that jet radiation is enhanced by traceable logarithms. This makes improved predictions for QCD observables based on resummation possible. The general strategy is to redefine the perturbative series from powers of $\alpha_{s}$ to including the relevant logarithms; the simplified structure of these enhanced terms then allows for a resummation to all orders. Once the resummed form is known we can match onto a fixed order calculation and avoid double-counting. For Sudakov-type logarithms a general method for this type of resummation is available [49], and for particular event shape observables an automated approach exists [50]. In LHC analyses, the resummation of finite logarithms in the presence of a jet-veto scale is of interest [24, 51].

A numerical approach to resummation is provided by parton-shower simulations [52]. It is automated in the multi-purpose Monte Carlo generators Pythia [53, 54], Herwig [55, 56] and Sherpa $[57,58]$ to leading order in the strong coupling combined with the resummation of leading collinear logarithms (LO/LL). This method differs from the previous approaches in that the full spectrum of final state partons or hadrons is produced explicitly. While the 
parton shower is well defined for relatively small transverse momenta of the jets it is not applicable for hard jet radiation. However, this limitation is overcome by the CKKW [16, 17], MLM [18], and CKKW-L [59, 60] jet-merging algorithms, that incorporate the treelevel matrix-element corrections for the first few hardest emissions [52, 61].

A complementary strategy is provided by the MC@NLO [62, 63] and POWHEG [6466] approaches, that realize the matching of NLO calculations with parton showers. While these methods guarantee NLO/LL accuracy only the first/hardest shower emission gets corrected by the real-emission matrix element. Higher jet multiplicities are described in the parton-shower approximation only. First attempts to combine the NLO/LL approaches with the tree-level merging ansatz have been reported recently [67, 68]. An unprecedented level of sophistication for predicting multi-jet final states is achieved by the promotion of merging algorithms to next-to-leading order accuracy [69-71].

Even though we can nowadays simulate multi-jet events, a detailed understanding of inclusive or exclusive $n_{\text {jets }}$ distributions at the LHC is still missing. Its universal features have been studied since 1985 [10-12]. Scaling patterns can be conveniently displayed in the ratio of successive exclusive jet cross-sections

$$
R_{(n+1) / n}=\frac{\sigma_{n+1}}{\sigma_{n}}=\frac{P_{n+1}}{P_{n}} \quad \text { with } \quad P_{n}=\frac{\sigma_{n}}{\sigma_{\text {tot }}} .
$$

We define the jet multiplicity $n$ as the number of jets in addition to the hard process, e.g. $\sigma_{1}$ for pure QCD di-jets is experimentally a 3-jet final state. Jets which are part of the hard process are not included in the scaling analysis because they do not arise from single QCD emissions.

Two patterns provide limiting cases for most LHC processes and are referred to as staircase and Poisson scaling. Staircase scaling is defined as constant ratios between the successive multiplicity cross sections

$$
R_{(n+1) / n}=R \equiv e^{-b},
$$

where $R$ and $b$ are constant. The exclusive $n$-jet rate for this distribution is $\sigma_{n}=\sigma_{0} e^{-b n}$ where $\sigma_{0}$ is the 0 -jet exclusive cross section. Staircase scaling for exclusive and inclusive jet rates is equivalent, with identical values of $R$. For a Poisson distribution with expectation value $\bar{n}$ the rates are

$$
P_{n}=\frac{\bar{n}^{n} e^{-\bar{n}}}{n !} \quad \text { or } \quad R_{(n+1) / n}=\frac{\bar{n}}{n+1} .
$$

More properties of the distributions are described in ref. [1].

Examples of Poisson [72] and staircase [73-79] scaling are found in current LHC analyses, although especially with the former, the examples are limited. Recent computations in fixed order perturbation theory [80] confirm that the staircase pattern improves at NLO compared to the LO prediction. In this paper we propose a number of different test-beds for both scaling behaviors, and provide predictions for the $n_{\text {jets }}$ ratios. In particular we suggest two explicit measurements based on simple extensions of already existing analyses, namely $Z+$ jets production and gap jet studies in pure QCD events. 
Establishing the origin of scaling in radiated jets from first principles is the primary purpose of this publication. The line of reasoning was touched on in our previous work, but a complete treatment is lacking. Besides constituting an aspect of perturbative QCD on its own, a fundamental understanding of scaling is obligatory for subsequent phenomenological applications. Only through an underlying mechanism can we convincingly argue that scaling patterns supplement the information provided by fixed-order or parton-shower calculations.

This paper is arranged as follows. In section 2 we introduce the standard theoretical framework for the parton shower, and elaborate on primary emissions with respect to the core process as a necessary condition for Poisson scaling. In conjunction, we demonstrate in the context of generating functionals that scaling patterns naturally emerge and can be proved to all multiplicities. In section 3 we generalize our discussion to hadron colliders. We introduce the corresponding generating functional formalism and discuss the effect of initial state PDFs, both through the kinematics and initial-state backward evolution. We close this section by showing that also in BFKL evolution the same scaling patterns appear as limiting cases. Having a firm understanding of the emergence of the scaling patterns from QCD, we can define cut regimes which exhibit idealized scaling. In section 4 we propose two experimental measurements where the underlying hypotheses of this paper can be tested at the LHC. We conclude and discuss possible extensions of our ideas in section 5 .

\section{$2 \quad$ Final state splittings}

Poisson statistics describe individual events occurring repeatedly and independently. For particle emissions this implies a non-trivial assumption. For successive photon radiation in QED it is the standard solution to divergences arising from soft and collinear photon radiation. The underlying approximation for multiple soft photon radiation is the eikonal approximation, in which the radiation of a soft photon does not affect the hard process. The eikonal approximation states nothing but a basic assumption of the Poisson process each successive photon sees the same unaltered hard process. For QCD it has been known for some time that the non-abelian nature of soft-gluon radiation leads to a deviation from Poisson statistics for gluon multiplicities [81]. When we measure jet ratios it is important to separate general exponentiation [82-86] from a Poisson distribution; to generate a Poisson distribution the exponentiation of soft real and virtual corrections has to include exactly the single-emission matrix element.

\subsection{Final-state parton shower}

The simulation of a LO/LL event in the parton shower approximation starts with the generation of a single phase space point for the partonic core process. The process' external (colored) lines then act as seeds for the subsequent parton shower evolution. Driven by unitarity they start at the hard process scale $Q$ and finish at the shower cutoff scale $Q_{0} \sim$ $1 \mathrm{GeV}$. Hard matrix element corrections for an arbitrary number of additional particles can be added to the parton shower using the above-mentioned matching schemes [16-18, 59, 60]. 
However, in this section we treat all additional emissions as coming from the parton shower and disregard matrix element corrections.

The basis of the LL parton shower is the fully factorized form of the collinear matrix element and phase space $d \sigma_{n+1} \sim d \sigma_{n} P_{1 \rightarrow 2} d \Phi_{1}$. Using this simplification the parton shower remains local, but loses information on spin, color correlations and interference effects in addition to higher order terms neglected in the $1 \rightarrow 2$ splitting kernels $P_{1 \rightarrow 2}$. Besides practicality, one of the benefits of collinear factorization is that the resummation of LL and some NLL contributions follow very naturally. To see this, we represent the evolution along an individual line by integrating the Sudakov factor over the appropriate virtuality scales from the lower cutoff $Q_{0}^{2}$ to a free hard scale $t$

$$
\Delta_{j}(t)=\exp \left[-\int_{Q_{0}^{2}}^{t} d t^{\prime} \Gamma_{j}\left(t, t^{\prime}\right)\right] .
$$

For the regularized splitting kernels $\Gamma_{j}$ we use the next-to-leading logarithmic approximation

$$
\Gamma_{j}\left(t, t^{\prime}\right)=c_{j} \frac{\alpha_{s}\left(t^{\prime}\right)}{2 \pi t^{\prime}}\left(\log \frac{t}{t^{\prime}}-A_{j}\right),
$$

with color factors $c_{j}=C_{F}\left(C_{A}\right)$ and the constant terms $A_{j}=3 / 2(11 / 6)$ for gluon emission off a quark (gluon). The lower cutoff scale $Q_{0}$ is omitted in the argument of the Sudakov form-factor. Expanding the exponential we see that eq. (2.1) represents an arbitrary number of soft and collinearly enhanced emissions, either resolved or unresolved.

To describe a parton-shower simulated event we note that the QCD evolution proceeds as an integration of the product Sudakov along the virtuality $t$,

$$
\boldsymbol{\Delta}(t)=\prod_{\text {ext lines }} \Delta_{j}(t) \equiv e^{-\boldsymbol{\Gamma}}
$$

The product defining $\boldsymbol{\Gamma}$ is over the appropriate factors for each external line, where $j$ denotes the particle flavor. Limiting ourselves to final state splittings this expression only contains evolution kernels as shown in eq. (2.1), and it is by construction guaranteed to exponentiate with an appropriate expression $\boldsymbol{\Gamma}$. As long as $\boldsymbol{\Gamma}$ is fully local and does not depend on previous emissions it is guaranteed to produce a Poisson distribution for the multiplicities. The exponentiated form in eq. (2.3) immediately identifies $\bar{n}=\boldsymbol{\Gamma}$. This statement does not depend on the form of $\boldsymbol{\Gamma}$ or its dependence on the hard scale $t$. All that matters is that each splitting does not change the subsequent evolution. In the remainder of this paper we define all emissions directly contained in the expansion of eq. (2.3) as primary with respect to the core process.

The first splitting in the parton shower picture defines the single emission probability. Following figure 1 a second emission can then appear from the original leg or off the first emission. For the former, this emission is contained in eq. (2.3) and does not change the Poisson pattern. The latter changes the exponential; we refer to it as secondary with respect to the original hard process. From a scaling perspective the relevant questions are 

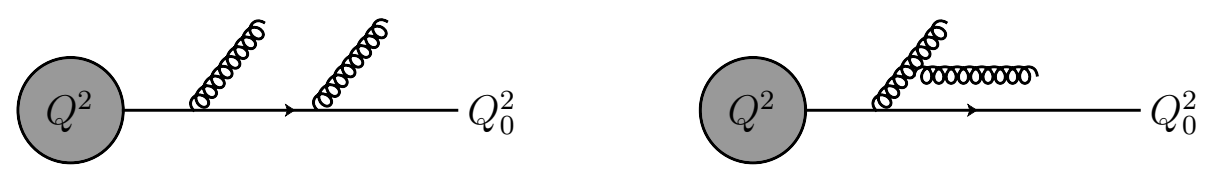

Figure 1. Simplest primary (left) and secondary contributions (right) assuming a core process with a hard quark line.

first, what is the relative size of the two contributions; and second if we can change the individual strengths of primary and secondary emissions through kinematic cuts.

In the parton shower approximation we can associate specific integrals over virtuality with individual partonic structures appearing in the final state evolution. An alternative evolution ordered in a consistent variable (e.g. angle) is logarithmically equivalent. Using this formalism the primary contribution to two gluon emission off a hard quark shown in figure 1 is

$$
\sigma^{\text {primary }}\left(Q^{2}, Q_{0}^{2}\right)=c^{\text {primary }} \int_{Q_{0}^{2}}^{Q^{2}} d t \Gamma\left(Q^{2}, t\right) \Delta_{g}(t) \int_{Q_{0}^{2}}^{Q^{2}} d t^{\prime} \Gamma\left(Q^{2}, t^{\prime}\right) \Delta_{g}\left(t^{\prime}\right) .
$$

The coefficient $c^{\text {primary }}$ which includes the Sudakovs associated with the hard line is process dependent, as this hard line can be either a quark or a gluon. The two external scales are the scale $Q$ of the hard process and the lower cutoff scale $Q_{0}$. If the primary emissions are strongly ordered in the evolution variable, the corresponding phase space factor $1 / 2$ is absorbed in $c^{\text {primary }}$. The simplest secondary contribution also shown in figure 1 is,

$$
\sigma^{\text {secondary }}\left(Q^{2}, Q_{0}^{2}\right)=c^{\text {secondary }} \int_{Q_{0}^{2}}^{Q^{2}} d t \Gamma\left(Q^{2}, t\right) \Delta_{g}(t) \int_{Q_{0}^{2}}^{t} d t^{\prime} \Gamma\left(t, t^{\prime}\right) \Delta_{g}\left(t^{\prime}\right)
$$

The splitting kernels in the two expressions only differ in the integral boundaries for the second emission. In the leading logarithmic approximation (in the exponent) for the Sudakov factors, we can perform the integrals in eq. (2.4) and eq. (2.5) in terms of error functions. The full expressions are not particularly enlightening, but two specific limits contain crucial information.

(1) $\frac{\alpha_{s}}{\pi} \log ^{2} \frac{Q}{Q_{0}} \gg 1$

In this limit we expand eq. (2.4) and eq. (2.5) around $Q_{0} / Q \rightarrow 0$ and find the leading terms

$$
\begin{aligned}
\sigma^{\text {primary }} & =\frac{c^{\text {primary }}}{4}\left[\frac{\alpha_{s}}{C_{A}} \log ^{2} \frac{Q}{Q_{0}}-\sqrt{\frac{4 \alpha_{s}}{C_{A}^{3}}} \log \frac{Q}{Q_{0}}+\mathcal{O}\left(\frac{Q_{0}^{2}}{Q^{2}}\right)\right] \\
\sigma^{\text {secondary }} & =\frac{c^{\text {secondary }}}{4}\left[(\sqrt{2}-1) \sqrt{\frac{\alpha_{s}}{C_{A}^{3}}} \log \frac{Q}{Q_{0}}+\mathcal{O}\left(\frac{Q_{0}^{2}}{Q^{2}}\right)\right] .
\end{aligned}
$$

Their ratio scales like $\sigma^{\text {primary }} / \sigma^{\text {secondary }} \propto \sqrt{\alpha_{s}} \log Q / Q_{0}$, i.e. the primary emissions are logarithmically enhanced. In the limit of a large logarithm (high single emission 
probability) the distribution of final state emissions are increasingly primary, and therefore give a Poisson distribution.

Physically interpreting eq. (2.6), a second logarithm in the secondary contribution would come from the right-most Sudakov of eq. (2.5). However, it has vanishing support for $Q \rightarrow \infty$ and does not appear in the approximate result. The emitted gluon in this case spans a vanishing relative fraction in virtuality space where it may emit an additional parton.

(2) $\frac{\alpha_{s}}{\pi} \log ^{2} \frac{Q}{Q_{0}} \ll 1$

Taking this limit of eq. (2.4) and eq. (2.5), we find

$$
\begin{aligned}
\sigma^{\text {primary }}\left(Q^{2}, Q_{0}^{2}\right) & =c^{\text {primary }} \frac{\alpha_{s}^{2}}{4(2 \pi)^{2}} \log ^{4} \frac{Q}{Q_{0}}+\mathcal{O}\left(\alpha_{s}^{3} \log ^{6} \frac{Q}{Q_{0}}\right) \\
& =6 \frac{c^{\text {primary }}}{c^{\text {secondary }}} \sigma^{\text {secondary }}\left(Q^{2}, Q_{0}^{2}\right) .
\end{aligned}
$$

The two contributions become logarithmically equivalent and differ by an $\mathcal{O}(1)$ constant depending primarily on color factors. In this regime the emission probability is small and the final state is selected democratically. The formerly Poisson scaling pattern receives large contributions from subsequent or secondary splittings. Note that to justify the logarithmic expansion we still require $\log ^{2} Q / Q_{0}>1$ but not large enough to spoil the small emission probability.

In appendix A we use a toy model of secondary splittings to show that $c^{\text {secondary }}$ gives the subsequent splitting parameter $\bar{n}^{\prime}$ in an iterated inhomogeneous Poisson distribution.

\subsection{Generating functional for jet fractions}

Following the argument presented in the last section we need a way to derive scaling patterns for arbitrarily high parton multiplicities. The generating functional formalism for QCD allows us to calculate resummed jet quantities [14, 15]. We construct a generating functional in an arbitrary parameter $u$ by demanding that repeated differentiation at $u=$ 0 gives exclusive multiplicity distributions. Different moments of the same generating functional then produce more inclusive jet observables. This gives us a set of coupled integral equations which we can solve in the limit of large and small emission probabilities. We will find that the derived jet multiplicity distributions follow a Poisson or staircase pattern, respectively.

For a known series of functions $P_{n}$ we define

$$
\Phi=\sum_{n=0}^{\infty} u^{n} P_{n-1} \quad \text { with } \quad P_{n-1}=\left.\frac{1}{n !} \frac{d^{n}}{d u^{n}} \Phi\right|_{u=0} .
$$

Note that for generating functionals we always suppress the argument $u$. In the application to gluon emission the explicit factor $1 / n$ ! corresponds to the phase space factor for identical bosons. The exclusive jet rates $P_{n}$ are defined in eq. (1.1). In accordance with that 
definition we only count radiated jets, for the generating functional that means we use $P_{n-1}$ instead of the usual $P_{n}$ consistently.

The quark and gluon generating functionals to next-to-leading logarithmic accuracy are

$$
\begin{aligned}
& \Phi_{q}\left(Q^{2}\right)=u \exp \left[\int_{Q_{0}^{2}}^{Q^{2}} d t \Gamma_{q}\left(Q^{2}, t\right)\left(\Phi_{g}(t)-1\right)\right] \\
& \Phi_{g}\left(Q^{2}\right)=u \exp \left[\int_{Q_{0}^{2}}^{Q^{2}} d t\left(\Gamma_{g}\left(Q^{2}, t\right)\left(\Phi_{g}(t)-1\right)+\Gamma_{f}(t)\left(\frac{\Phi_{q}^{2}(t)}{\Phi_{g}(t)}-1\right)\right)\right] .
\end{aligned}
$$

The splitting kernels are defined in eq. (2.2); gluon splitting to quarks, described by the kernel $\Gamma_{f}(t)$, is suppressed by a power of the logarithm. Assuming $Q \gg Q_{0}$, or a high emission probability as discussed in section 2.1, the largest contribution to the $t$ integration comes from the region where $t \approx Q_{0}^{2}$ and $\Phi_{q, g}(t) \approx \Phi_{q, g}\left(Q_{0}^{2}\right) \approx u$. Both evolution equations then read

$$
\Phi_{j}\left(Q^{2}\right)=u \exp \left[\int_{Q_{0}^{2}}^{Q^{2}} d t \Gamma_{j}\left(Q^{2}, t\right)(u-1)\right]=\frac{u \Delta_{j}\left(Q^{2}\right)}{\Delta_{j}\left(Q^{2}\right)^{u}}
$$

with the Sudakov factor defined in eq. (2.1). For the jet rates we find a Poisson distribution

$$
P_{n-1}=\Delta_{j}\left(Q^{2}\right) \frac{\left|\log \Delta_{j}\left(Q^{2}\right)\right|^{n-1}}{(n-1) !} \quad \text { or } \quad R_{(n+1) / n}=\frac{\left|\log \Delta_{j}\left(Q^{2}\right)\right|}{n+1} .
$$

Again, the jet counting reflects our convention that of $n$ jets only $n-1$ are radiated off the hard line. The result in eq. (2.11) reflects the same underlying physics as eq. (2.6), namely a universal logarithmic enhancement of the primary emission over subsequent ones. The latter also covers the sub-leading terms to the pure Poisson distribution, and thus determines the size of the leading corrections to eq. (2.11). For a hard quark as well as for a hard gluon line this Poisson distribution contains only logarithmically enhanced gluon radiation. According to the approximation of eq. (2.10), any subsequent splitting of the radiated gluons is subleading.

To investigate deviations from this perfect Poisson pattern we first study jet fractions $P_{n}$ for up to four emissions in $e^{+} e^{-}$collisions. We analytically derive them using the generating functional, eq. (2.8), and show the results in appendix B. Expanding them to $\mathcal{O}\left(\alpha_{s}^{5}\right)$ and combining this with inclusive unitarity $\sum P_{n}=1$ at each fixed order gives the double-logarithmically enhanced contribution for $n \leq 5$. They show the expected Poisson pattern for the abelian terms $\propto C_{F}$,

$$
P_{n}=\frac{\left[\Delta_{q}\left(Q^{2}\right)\right]^{2}}{n !}\left(\int_{Q_{0}^{2}}^{Q^{2}} d t \Gamma_{q}\left(Q^{2}, t\right) \Delta_{g}(t)\right)^{n} .
$$

The additional gluon Sudakov compared to eq. (2.11) takes into account that the radiated gluons do not split in the primary contributions. The non-abelian terms $\propto C_{A}$ do not exponentiate with respect to the $q \bar{q}$ final state. They deviate from Poisson scaling starting at two additional jets and provide sensitivity to the triple-gluon vertex [87, 88]. The 
secondary contribution to two-jet emission is color enhanced via $C_{A} / C_{F}$, but ultimately smaller than the Poisson term due to the averaging factor over the second splitting function. For the leading double logarithm this is a simple suppression factor of $1 / 6$. In appendix A we compare the LL jet rates and a toy model for an iterated Poisson process.

In addition to purely non-abelian splittings, mixed primary and secondary contributions also deviate from the Poisson pattern. This effect we can study in the average jet multiplicity in $e^{+} e^{-}$collisions. This observable conveniently singles out all emission histories which are secondary with respect to the core process. This way we generate the highest non-abelian terms at each perturbative order,

$$
\begin{aligned}
\left\langle n_{\text {jets }}\right\rangle= & 2+\frac{\alpha_{s}}{\pi} \frac{C_{F}}{2} \log ^{2} y_{\text {cut }} \\
& +\left(\frac{\alpha_{s}}{\pi}\right)^{2} \frac{C_{A} C_{F}}{48} \log ^{4} y_{\text {cut }}+\left(\frac{\alpha_{s}}{\pi}\right)^{3} \frac{C_{A}^{2} C_{F}}{2880} \log ^{6} y_{\text {cut }} \\
& +\left(\frac{\alpha_{s}}{\pi}\right)^{4} \frac{C_{A}^{3} C_{F}}{322560} \log ^{8} y_{\text {cut }}+\left(\frac{\alpha_{s}}{\pi}\right)^{5} \frac{C_{A}^{4} C_{F}}{58060800} \log ^{10} y_{\text {cut }}+\mathcal{O}\left(\left(\frac{\alpha_{s}}{\pi}\right)^{6} \log ^{12} y_{\text {cut }}\right) .
\end{aligned}
$$

The second term gives the Poisson distribution for abelian emissions off the quark line, i.e. $\bar{n}$ as given in eq. (2.12). All other terms are leading in $C_{A}$ for a given power of $\alpha_{s}$, i.e. they originate from a single radiated gluon [15]. Mixed terms of order $C_{F}^{n+1} C_{A}^{m}$ are absorbed into purely $C_{F} C_{A}^{m}$ terms through exponentiation. Therefore, the combination $\left\langle n_{\text {jets }}\right\rangle-2-\alpha_{s} C_{F} /(2 \pi) \log ^{2} y_{\text {cut }}$ reflects the non-Poisson nature of the purely secondary emission at leading logarithm.

Going back to the limiting cases introduced in section 2.1 we also find a recursive solution for small emission probabilities when restricting ourselves to pure Yang-Mills theory. We take $Q \sim Q_{0}$, but large enough to define logarithmically enhanced terms. The generating functional for a theory with only gluons satisfies

$$
\begin{aligned}
\frac{d \Phi_{g}\left(Q^{2}\right)}{d Q^{2}}= & \Phi_{g}\left(Q^{2}\right) \frac{C_{A}}{2 \pi Q^{2}}\left[-\frac{11 \alpha_{s}\left(Q^{2}\right)}{6}\left(\Phi_{g}\left(Q^{2}\right)-1\right)+\int_{Q_{0}^{2}}^{Q^{2}} d t \frac{\alpha_{s}(t)}{t}\left(\Phi_{g}(t)-1\right)\right] \\
= & \Phi_{g}\left(Q^{2}\right) \\
& \times\left[\tilde{\Gamma}_{g}\left(Q^{2}, Q_{0}^{2}\right)\left(\Phi_{g}\left(Q^{2}\right)-1\right)-\frac{C_{A}}{2 \pi Q^{2}} \int_{Q_{0}^{2}}^{Q^{2}} d t \log \frac{t}{Q_{0}^{2}}\left(\frac{d}{d t} \alpha_{s}(t)\left(\Phi_{g}(t)-1\right)\right)\right],
\end{aligned}
$$

after integrating by parts and defining

$$
\tilde{\Gamma}_{g}\left(Q^{2}, Q_{0}^{2}\right)=C_{A} \frac{\alpha_{s}\left(Q^{2}\right)}{2 \pi Q^{2}}\left(\log \frac{Q^{2}}{Q_{0}^{2}}-\frac{11}{6}\right) .
$$

Only including the leading logarithms this is simply the negative splitting function from eq. (2.2), but it obviously differs beyond this approximation. To establish the accuracy of eq. (2.14) including the maximal amount of NLL contributions, we expand in powers of 


$$
\begin{aligned}
& \epsilon \equiv\left(Q^{2}-Q_{0}^{2}\right) / Q^{2} \\
& \tilde{\Gamma}_{g}\left(Q^{2}, Q_{0}^{2}\right)\left(\Phi_{g}\left(Q^{2}\right)-1\right) \approx \frac{C_{A} \alpha_{s}}{2 \pi Q^{2}}\left[-\frac{11}{6}+\left(2-\frac{11 \alpha_{s} b_{0}}{3 \pi}\right) \epsilon+\mathcal{O}\left(\epsilon^{2}\right)\right] \\
& \frac{C_{A}}{2 \pi Q^{2}} \int_{Q_{0}^{2}}^{Q^{2}} d t \log \frac{t}{Q_{0}^{2}}\left(\frac{d}{d t} \alpha_{s}(t)\left(\Phi_{g}(t)-1\right)\right) \approx-\frac{C_{A} \alpha_{s}}{2 \pi Q^{2}}\left[-4 \epsilon^{2}+\mathcal{O}\left(\epsilon^{3}\right)\right] .
\end{aligned}
$$

The logarithmic and finite contribution from the first term on the r.h.s. of the differential equation are of order $\epsilon^{1}$ and $\epsilon^{0}$ while the formally infinite series from the second term in eq. (2.14) starts at $\epsilon^{2}$. Keeping only terms linear in $\epsilon$ we obtain the simple form

$$
\frac{d \Phi_{g}\left(Q^{2}\right)}{d Q^{2}} \approx \Phi_{g}\left(Q^{2}\right) \tilde{\Gamma}_{g}\left(Q^{2}, Q_{0}^{2}\right)\left(\Phi_{g}\left(Q^{2}\right)-1\right)
$$

Including the boundary condition $\Phi_{g}\left(Q_{0}^{2}\right)=u$ we can solve this,

$$
\Phi_{g}\left(Q^{2}\right)=\frac{1}{1+\frac{(1-u)}{u \tilde{\Delta}_{g}\left(Q^{2}\right)}} \quad \text { with } \quad \tilde{\Delta}_{g}\left(Q^{2}\right)=\exp \left[-\int_{Q_{0}^{2}}^{Q^{2}} d t \tilde{\Gamma}_{g}\left(t, Q_{0}^{2}\right)\right]
$$

Neglecting the effects of the running coupling, $\tilde{\Delta}_{g}\left(Q^{2}\right)$ is a Sudakov form factor. Including the running coupling, eq. (2.18) differs from the standard Sudakov in eq. (2.1) starting at higher orders,

$$
\frac{\tilde{\Delta}_{g}\left(Q^{2}\right)}{\Delta_{g}\left(Q^{2}\right)}=\exp \left(-\frac{\alpha_{s}^{2}}{12 \pi} b_{0} \log ^{3} \frac{Q^{2}}{Q_{0}^{2}}\right) .
$$

Taking derivatives of the generating functional in eq. (2.18) at $u=0$ we can compute the exclusive jet rates

$$
P_{n-1}=\tilde{\Delta}_{g}\left(Q^{2}\right)\left(1-\tilde{\Delta}_{g}\left(Q^{2}\right)\right)^{n-1} \quad \text { or } \quad R_{(n+1) / n}=1-\tilde{\Delta}_{g}\left(Q^{2}\right) .
$$

These constant ratios define a staircase pattern. Comparing eq. (2.11) and eq. (2.20) we see that in two distinct phase space regimes we find two clear scaling patterns for the Yang-Mills or pure gluon case. Both of them can arise in final state gluon radiation, which means they should in principle be observable in $e^{+} e^{-} \rightarrow$ jets events.

The all-order theoretical predictions for Poisson scaling, eq. (2.11), and staircase scaling, eq. (2.20), we can compare to simulated $e^{+} e^{-} \rightarrow$ jets events. To cover both, a large scale separation $Q \gg Q_{0}$ as well as a democratic scale $Q \sim Q_{0}$, we use a large centerof-mass energy of $2 \mathrm{TeV}$ and a very small lower cutoff $y_{\text {cut }}=5 \cdot 10^{-7}$ for the Durham jet-reconstruction algorithm [89]. In figure 2 we show jet ratios $R_{(n+1) / n}$ for a large range of $n$. Indeed, we observe Poisson as well as staircase scaling. The same behavior is known from hadron colliders for example in $p p \rightarrow \gamma+$ jets production [13]: for relatively low $n$ values the emission is dominated by large scale differences, inducing a Poisson pattern. For large jet multiplicity individual emissions are not affected by a large scale difference, so we see a staircase tail. While this transition is a solid QCD prediction it has not been studied experimentally (yet). 


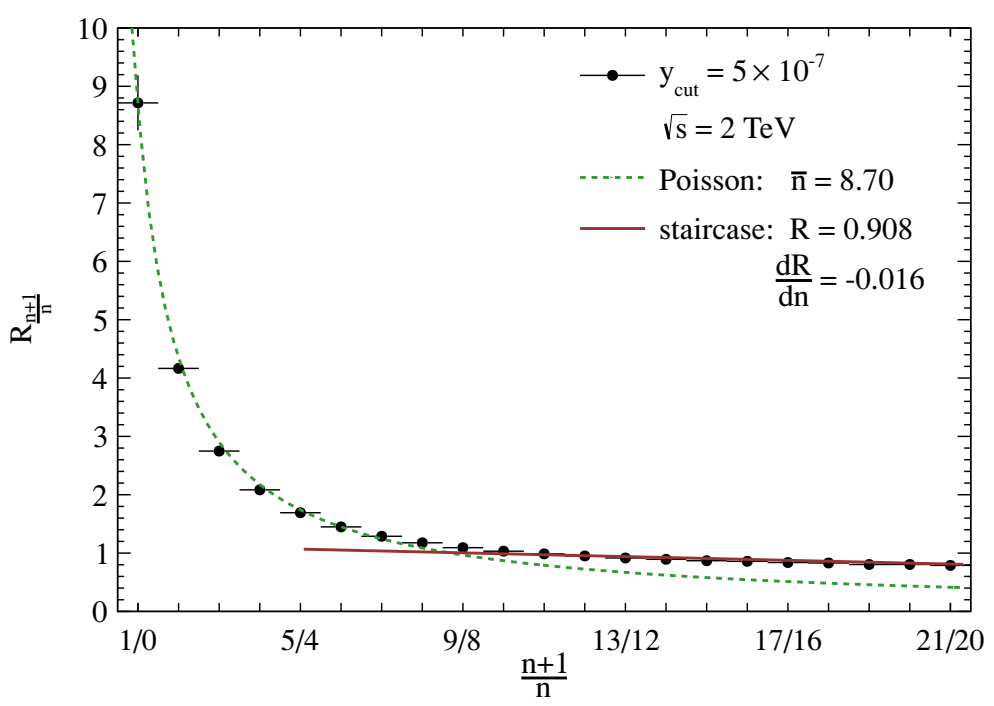

Figure 2. Jet ratios $R_{(n+1) / n}$ in $e^{+} e^{-} \rightarrow$ jets production at $2 \mathrm{TeV}$ center-of-mass energy. We show a Poisson fit with $\bar{n}=8.7$ and a staircase fit to the tail. We use Sherpa $[57,58,90]$ with the $g \rightarrow q \bar{q}$ shower splittings switched off.

\subsection{Matrix element corrections}

In all of the above discussion we only assume logarithmically induced emission and neglect any kind of phase space effects. A simple test case for the relative contributions of primary vs subsequent emissions including additional phase space information is two-gluon emission from a $q \bar{q}$ dipole. The squared matrix element for strongly ordered two-gluon emissions is $[91-93]$

$$
\left|\mathcal{M}\left(p_{1}, p_{2}\right)\right|^{2}=\frac{32 C_{F}}{p_{T, 1} p_{T, 2}}\left[C_{A}\left(\frac{\cosh \left(\eta_{1}-\eta_{2}\right)}{\cosh \left(\eta_{1}-\eta_{2}\right)-\cos \left(\phi_{1}-\phi_{2}\right)}-1\right)+2 C_{F}\right],
$$

where $\eta_{i}$ are the gluon rapidities, $\phi_{i}$ are the azimuthal angles and $p_{T, i}$ the transverse momenta. The term proportional to $C_{F}$ with its very simple kinematic structure represents the primary emissions. The $C_{A}$ term corresponds to the subsequent emission contribution and contains an interesting dependence on the phase space.

The transverse momentum integration leads to $\log Q / Q_{0}$ terms for both contributions, which we now assume to not be too large - otherwise we would be logarithmically dominated and the results from the previous section would apply. Instead, we are interested in the $\eta$ and $\phi$ dependence. The question is whether such final state kinematics can be observed as deviations from our scaling patterns.

In figure 3 we examine the primary and subsequent contribution as a function of $\Delta \eta$. Testing different $\Delta \phi$ values, we see that the secondary emission dominates when the gluons are close. For $\Delta \phi=\pi / 2$ the $C_{A}$ term vanishes for all rapidity separations. Note that for $\sqrt{(\Delta \eta)^{2}+(\Delta \phi)^{2}} \leq R$ the two emissions are clustered in a single jet of radius $R$ (typically $R \approx 0.4-0.7)$ and as such do not contribute to the 4 -jet rate. This suggests that for large angle emissions the $n_{\text {jets }}$ spectrum will remain Poisson even including matrix element 




Figure 3. Contribution from secondary emission to the squared matrix elements given in eq. (2.21) as a function of $\Delta \eta$ for $\Delta \phi=0, \pi / 6, \pi / 4, \pi / 3$ versus the constant primary contribution (horizontal line). All curves are normalized to $K=64 C_{F}^{2} /\left(p_{T, 1} p_{T, 2}\right)$.

information. Or in other words subsequent emissions breaking the Poisson pattern are unlikely to be widely separated.

Although eq. (2.21) contains particular matrix element information, it is still based on the eikonal approximation and does not include kinematical constraints. However, we know that subsequent emissions lead to deviations from Poisson scaling in all phase space regions. In order to get a handle exclusively on effects from outside the soft or soft-collinear regime, we consider the photon multiplicities in the QED process $e^{+} e^{-} \rightarrow e^{+} e^{-}+n \gamma$. To leading order all photon emissions are primary.

We consider the cross sections for the processes $e^{+} e^{-} \rightarrow e^{+} e^{-}+n \gamma$ at $\sqrt{s}=500 \mathrm{GeV}$ using the exact tree-level matrix elements regulated using the $k_{T}$ measure

$$
\frac{2 \min \left(E_{i}^{2}, E_{j}^{2}\right)}{s}\left(1-\cos \theta_{i j}\right)>y_{\text {cut }} \text {. }
$$

For small values of $y_{\text {cut }}$ we should find a Poisson pattern in the exclusive photon rates, which we confirm in figure 4. For larger $y_{\text {cut }}$ the different multiplicity distributions start deviating from the Poisson pattern. The ratios are pushed apart from one another, opposite to what we expect from a staircase pattern. The reason is that each emission takes a non-negligible amount of the total energy of the event and suppresses the phase space for subsequent emissions. Going back to the two main scaling patterns this means that matrix element and final-state phase space effects are not responsible for the transition from Poisson to staircase scaling.

\section{Hadron colliders}

The analytic form of the fixed energy jet fractions given in appendix B indicates that a final state cascade initiated by a $q \bar{q}$ pair follows neither a Poisson nor a staircase scaling 


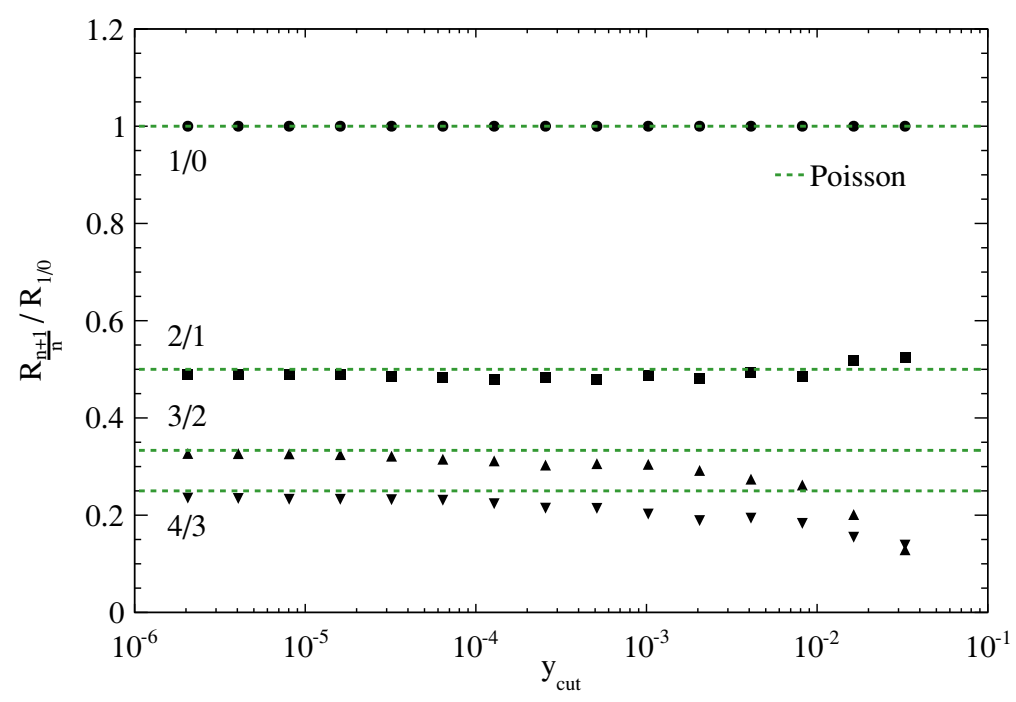

Figure 4. Normalized ratios for the photon multiplicity in $e^{+} e^{-} \rightarrow e^{+} e^{-}+n \gamma$ as a function of the resolution parameter $y_{\text {cut }}$. The solid lines correspond to the perfect Poisson hypothesis. We use Sherpa $[57,58]$.

pattern at low multiplicities. However, from $Z+$ jets production we know that essentially all jet ratios are constant, with an even stronger suppression of $R_{1 / 0}$ [94]. This suggests that additional effects drive the jet ratios at hadron colliders towards a staircase pattern. One possible cause is that incoming partons do not on average carry the same energy fractions $x$ for different final state jet multiplicities; in that case we might observe an initial-state phase space effect. Second, jets at hadron colliders are typically generated through initial state radiation, and we know that the initial-state parton shower behaves somewhat differently from final state splittings.

\subsection{Generating functional for incoming hadrons}

The basis of the QCD treatment of hadron collider physics is collinear factorization which allows us to employ the generating functional method $[14,15,20,21]$. Before we can apply any of this to jet counting we need to clarify our choice of the factorization scale $\mu_{F}$ in exclusive $n_{\text {jet }}$ rates, i.e. in the presence of a jet-counting or jet-veto scale $p_{V}$. The resummation properties of the DGLAP equation identify the combined renormalization and factorization scale with a collinear cutoff below which initial state splittings are unresolved and influence only the functional dependence on the partonic energy fraction $x$. Because we are interested in radiated jets with $p_{T} \geq p_{V}$ we identify the factorization and the jet-veto scale, i.e. $\mu_{F} \equiv p_{V}$. Note that this choice furthermore avoids generating additional finite though potentially large logarithms in the ratio $\mu_{F} / p_{V}$ [24].

Symbolically, going from final state radiation in $e^{+} e^{-}$collisions to deep inelastic scattering (DIS) with initial state radiation and parton densities we replace the two generating functionals, distinguishing time-like from space-like splittings,

$$
\Phi_{q}\left(Q^{2}, p_{V}^{2}\right) \times \Phi_{\bar{q}}\left(Q^{2}, p_{V}^{2}\right) \quad \rightarrow \quad \Phi_{q / \bar{q}}\left(Q^{2}, p_{V}^{2}\right) \times \mathcal{Z}_{q / \bar{q}}\left(x, Q^{2}, p_{V}^{2}\right) .
$$


As in section 2.2 we omit the argument $u$ in all generating functionals. In the original DIS context all scales are defined in terms of the $e^{+} e^{-}$Durham algorithm [20,21], most notably the hard scale $Q$ and $p_{V} \equiv \mu_{F}$ as well as the softer resolution scale $Q_{0} \leq \mu_{F}$. We identify all three relevant scales $Q_{0}=\mu_{F}=p_{V}$. For the DIS analysis this corresponds to not further resolving the original macro-jets which define the separation of resolved jets and beam jets $[20,21]$. Again, this choice omits potentially large finite scale logarithms in our perturbative treatment.

We also introduce an explicit $x$ dependence in the generating functional for incoming partons as it is clear that PDF effects alter the possibility to radiate jets. Each emission takes away an energy fraction $1-z$ of the emitter; the $x$ value has to change correspondingly and splitting between different partons needs to be taken into account. From the factorization theorem we know that PDFs and partonic cross-sections also factorize at the generating functional level,

$$
\mathcal{Z}_{a}\left(x, Q^{2}, p_{V}^{2}\right)=\sum_{b} \int_{x}^{1} \frac{d z}{z} f_{b}\left(\frac{x}{z}, p_{V}^{2}\right) \mathcal{Z}_{a}^{b}\left(z, Q^{2}, p_{V}^{2}\right) .
$$

The parton densities we consistently evaluate at the scale $p_{V}$. This way, logarithmically enhanced parton splittings above $p_{V}$ are described by the partonic generating functional $\mathcal{Z}_{a}^{b}$. For the generating functional in DIS we start with a time-like generating functional for a single (anti-)quark and weight it with the proper electromagnetic coupling [20, 21]

$$
\Phi_{\mathrm{DIS}}=\sum_{a} e_{a}^{2} \Phi_{a}\left(Q^{2}, p_{V}^{2}\right) \mathcal{Z}_{a}\left(x, Q^{2}, p_{V}^{2}\right)
$$

The partonic cross-sections and jet evolution are the same for the quark and the anti-quark, but the PDFs are different. In DIS the final state kinematics fix $x$. Additional jets radiated off the incoming parton imply that in this case we probe higher $x$ values as given by the convolution in eq. (3.2).

The task is to find the evolution equations for $\mathcal{Z}_{a}^{b}$. To leading logarithm (LL) this turns out to be relatively simple. In the soft and collinear limit $[14,15]$ the eikonal approximation implies $z \approx 1$. Furthermore, the $g \rightarrow q \bar{q}$ splitting is logarithmically suppressed compared to the other splittings, so we can neglect it. Under these two LL assumptions the evolution equation and the corresponding generating function in eq. (3.2) read [20, 21]

$$
\begin{aligned}
& \mathcal{Z}_{a}^{b}\left(z, Q^{2}, p_{V}^{2}\right)=\delta(1-z) \delta_{a}^{b} \Phi_{a}\left(Q^{2}, p_{V}^{2}\right) \\
& \mathcal{Z}_{a}\left(x, Q^{2}, p_{V}^{2}\right)=f_{a}\left(x, p_{V}^{2}\right) \Phi_{a}\left(Q^{2}, p_{V}^{2}\right) .
\end{aligned}
$$

The PDF effects and the jet generating function factorize in $x$, so we can treat them independently. In general, $\Phi_{a}$ is a two-scale generating functional [20, 21, 95]. Because we identify $Q_{0}=p_{V}$ the second scale is suppressed and its evolution equation is almost the same as in the $e^{+} e^{-}$case. To leading logarithm we find

$$
\Phi_{a}\left(Q^{2}, p_{V}^{2}\right)=\exp \left[\int_{p_{V}^{2}}^{Q^{2}} d t \Gamma_{a}\left(Q^{2}, t\right)\left(\Phi_{g}\left(t, p_{V}^{2}\right)-1\right)\right] .
$$


Compared to eq. (2.9) the factor $u$ in front of the exponential is missing. The reason is that we cannot resolve a jet if there is not at least one space like splitting. The hard parton cannot produce a final state jet, so we always find the normalization condition $\Phi_{a}\left(p_{V}^{2}, p_{V}^{2}\right) \equiv 1$. The further evolution of emitted partons we describe with the time-like functional of eq. (2.9).

Moving on to Drell-Yan production with two incoming partons we need to replace the generating functionals, symbolically written, to

$$
\mathcal{Z}_{q / \bar{q}}\left(x_{a}, Q^{2}, p_{V}^{2}\right) \times \mathcal{Z}_{\bar{q} / q}\left(x_{b}, Q^{2}, p_{V}^{2}\right) .
$$

Thus, we replace the remaining time-like generating functional with a space-like generating functional to describe two incoming partons. A major complication is that the final state phase space does not fix $x_{a, b}$ anymore. Instead, we have to integrate over their allowed ranges and find the generating functional for the Drell-Yan process,

$$
\begin{aligned}
\Phi_{\text {Drell-Yan }} & =\sum_{a, b} \int d x_{a} d x_{b} \mathcal{Z}_{a}\left(x_{a}, Q^{2}, p_{V}^{2}\right) \mathcal{Z}_{b}\left(x_{b}, Q^{2}, p_{V}^{2}\right) \\
& \approx \sum_{a, b} \int d x_{a} d x_{b} f_{a}\left(x_{a}, p_{V}^{2}\right) f_{b}\left(x_{b}, p_{V}^{2}\right) \Phi_{a}\left(Q^{2}, p_{V}^{2}\right) \Phi_{b}\left(Q^{2}, p_{V}^{2}\right) \\
& \approx \sum_{a, b} f_{a}\left(x^{(0)}, p_{V}^{2}\right) \Phi_{a}\left(Q^{2}, p_{V}^{2}\right) f_{b}\left(x^{(0)}, p_{V}^{2}\right) \Phi_{b}\left(Q^{2}, p_{V}^{2}\right) .
\end{aligned}
$$

From this generating functional we can derive the individual $n$-jet rates. For the second line of eq. (3.7) we use the leading logarithmic approximation as in the DIS case. To arrive at the third line we replace the variable $x_{a, b}$ values by a typical partonic energy scale $x^{(0)}$. For typical hadron colliders processes we assume this value to be close to threshold and equal for the two incoming partons. The argument $u$ which generates the different $n$-jet rates is carried only by the generating functionals $\Phi_{a, b}\left(Q^{2}, p_{V}^{2}\right)$. Starting with two generating functionals for the two initial state particles, hard jet radiation with $p_{T}>p_{V}$ indeed factorizes from a PDF factor.

One apparent contradiction related to the PDF kinematics we need to resolve. On the one hand, in eq. (3.4) the eikonal approximation allows us to set $z \approx 1$, which means that the entire energy dependence is encoded in the PDF factor. On the other hand, each resolved jet requires a finite $p_{T}>p_{V}$. Hence, the integration range for $x_{a, b}$ is determined by the partonic $n$-jet process and $x^{(0)}$ implicitly depends on $u$. This implicit dependence we have to account for by hand. In particular for parton density regimes which increase towards small $x$ the majority of multi-jet events at the LHC are produced at threshold. The threshold value for any of the $n$-jet production rates we denote as $x^{(n)}$, leading us to the modified factorized form

$$
\Phi_{\text {Drell-Yan }}=\sum_{a, b} f_{a}\left(x^{(n)}, p_{V}^{2}\right) \Phi_{a}\left(Q^{2}, p_{V}^{2}\right) f_{b}\left(x^{(n)}, p_{V}^{2}\right) \Phi_{b}\left(Q^{2}, p_{V}^{2}\right) .
$$

We emphasize that $n$ is determined a posteriori upon differentiation with respect to $u$, so is presented for illustrative purposes only. Eq. (3.8) means that to leading logarithm the 
jet radiation pattern in the Drell-Yan case is the same as in $e^{+} e^{-} \rightarrow$ jets processes, modulo explicit PDF factors estimated using an $n$-dependent threshold kinematics. A similar approach can account for energy momentum conservation in soft-gluon resummation [96]. This way we leave the LL evolution of jets untouched and instead shift the $x$ value in the PDFs to account for additional jets. All our findings from section 2 we can immediately apply, once we understand the PDF correction factor in the next section.

\subsection{Parton density suppression}

In section 3.1 we have learned that to leading logarithmic accuracy the effects of the parton densities and jet emission factorize. For large jet multiplicities this explains the observed staircase scaling at hadron colliders [10-12, 73-79]. Parton densities contribute to this effect in particular at low multiplicities. When increasing the jet multiplicity the typical partonic energy fractions $x$ probed by the partonic process increase as well. The relative increase in $x$ is largest for low jet multiplicities.

In terms of the assumed threshold kinematics adding a jet with finite transverse momentum implies $x^{(n+1)}>x^{(n)}$. To compute the relative cost of producing an additional jet we estimate the ratio of PDF values evaluated at $x^{(n)}$ and $x^{(n+1)}$ as a function of the number of extra jets $n$. In effect this is the discretized second derivative with respect to $x$. For hadron collider processes involving two parton densities $f(x, Q)$ we define the PDF correction factor to the ratio of successive jet ratios $R_{(n+1) / n} / R_{(n+2) /(n+1)}$

$$
B_{n}=\left|\frac{\frac{f\left(x^{(n+1)}, Q\right)}{f\left(x^{(n)}, Q\right)}}{\frac{f\left(x^{(n+2)}, Q\right)}{f\left(x^{(n+1)}, Q\right)}}\right|^{2} .
$$

The square in the definition of $B_{n}$ reflects the two PDFs in hadron collisions. If for example the partonic ratio of two successive jet ratios is $R_{(n+1) / n} / R_{(n+2) /(n+1)} \sim c$ then the proper hadronic ratio becomes $B_{n} c$. We fix $Q$ for simplicity, but this only mildly affects our results.

The main effects are, first, that $B_{n}<1$ in most cases. This way PDF effects suppress the lower multiplicity ratios $R_{(n+1) / n}$. For large jet multiplicities the relative impact of yet another jet becomes small, $B_{n} \rightarrow 1$. The hadronic initial-state effect on the jet scaling disappears and we are back to the staircase pattern. Second, the PDF effect is largest for the steep gluon densities, as compared to the flatter valence quarks. Finally, allowing for variable $Q$ the PDF values $f(x, Q)$ increase (decrease) with $Q$ for low (high) $x$, with a cross-over point around $x \sim 0.1$. For small $x$ values the initial state evolution then suppresses jet ratios at high multiplicity or large $Q^{2}$.

What we are most interested in are PDF effects for the Drell-Yan process at lower multiplicities. We consider the threshold values $x^{(n)}$, for example for producing an on-shell $Z$-boson and one additional jet,

$$
x^{(1)}=\frac{\sqrt{m_{Z}^{2}+2\left(p_{T} \sqrt{p_{T}^{2}+m_{Z}^{2}}+p_{T}^{2}\right)}}{2 E_{\text {beam }}} .
$$





Figure 5. Left panel: estimated PDF suppression for inclusive (solid) and jet-associated (dashed, $p_{T}^{\text {lead }} \geq 100 \mathrm{GeV}$ ) Drell-Yan kinematics. We assume an initial state with $d$-quarks only. Right panel: same for Higgs production in gluon fusion with $m_{H}=125 \mathrm{GeV}$. The uncertainty encompasses two representative kinematical limits of the multi-jet final state, described in the text.

where $E_{\text {beam }}=3500 \mathrm{GeV}$ for the LHC in 2011. Comparing $x^{(1)}$ with $x^{(0)} \approx m_{Z} /\left(2 E_{\text {beam }}\right)$ shows a sizeable shift. For the two-jet threshold $x^{(2)}$ two limiting cases are either the additional jet adding merely $p_{T} /\left(2 E_{\text {beam }}\right)$ to $x^{(1)}$ or two approximately collinear jets recoiling against a hard $Z$. The variation between these two cases estimates the uncertainty on our method which can be generalized straightforwardly to the $n$-jet final state.

In the left panel of figure 5 we display $B_{n}$ for the estimated Drell-Yan kinematics, assuming each jet has transverse momentum $p_{T}=p_{V}=30 \mathrm{GeV}$. The effect on the first jet ratios is large, but quickly diminishes towards higher $n$. We also see that if we require a leading jet with large transverse momentum, $p_{T}^{\text {lead }} \geq 100 \mathrm{GeV}$, we move to sufficiently high $x$ such that additional jet ratios are unaffected by the PDF effect. It is reassuring to see that if we combine the PDF suppression of $R_{1 / 0}(0.46-0.65)$ with the $C_{A}$ enhancement of $R_{2 / 1}(1.36)$ and assume an original Poisson scaling we find $R_{1 / 0} / R_{2 / 1}=(0.67-0.95)$, in nice agreement with ATLAS data [94]. This beautifully illustrates that staircase scaling at large multiplicities can be derived from first principles QCD while for small multiplicities it is something like a sweet spot.

As an additional check, we present the PDF suppression in gluon-fusion Higgs production in the right panel of figure 5 . We assume $m_{H}=125 \mathrm{GeV}$, ignore flavor changes and consider jets with $p_{T}=p_{V}=30 \mathrm{GeV}$. The gluon PDF drops more rapidly for increasing $x$, inducing a large PDF suppression. On the other hand, the increasing energy of the core process as compared to the Drell-Yan process slightly decreases the effect. The combination of the two gives remarkably similar results to the Drell-Yan process.

\subsection{Initial-state parton shower}

As indicated above, jet radiation at hadron colliders is generated mostly through initial state radiation, which means that our final-state analysis of section 2 should be modified. 




Figure 6. Normalized ratios of gluon production rates originating from the backward evolution of $d$-quarks according to eq. (3.11). We assume different initial values for $x^{(0)}$. Each splitting is restricted to $z$ values generating a minimal increase of $\Delta x=0.02$. The dashed line indicates the expectation for a perfect Poisson distribution of the underlying jet rates.

We need to compute the spectrum of jets arising as primary emission in the backward evolution and acting as seeds for subsequent final-state radiation. For simplicity we just consider the backward evolution along an initial-state quark line with a single type of branching, namely gluon emission. The evolution proceeds through the space-like Sudakov form factor

$$
\Pi\left(t_{1}, t_{2} ; x\right)=\exp \left\{-\int_{t_{1}}^{t_{2}} \frac{d t}{t} \int \frac{d z}{z} \frac{\alpha_{s}}{2 \pi} P_{q \rightarrow q g}(z) \frac{f_{q}(x / z, t)}{f_{q}(x, t)}\right\},
$$

cf. ref. [97], with the appropriate splitting kernels for gluon emission $P_{q \rightarrow q g}$ and ignoring potential initial-state flavor changes here. The evolution of each initial state parton starts with momentum fraction $x_{i}$, determined by the hard process, and virtuality $t=x_{1} x_{2} S$, it terminates at the hadronic scales $x \approx 1$ and $t=Q_{0}^{2}$ associated with the transition to non-perturbative physics.

We know from section 2 that a single time-like Sudakov form factor produces perfect Poisson scaling. Indeed, whenever we have a non-emission probability represented by $e^{-\boldsymbol{\Gamma}}$ as in eq. (2.3), where $\boldsymbol{\Gamma}$ does not change as a result of a splitting, the process is guaranteed to produce Poisson scaling. For backwards evolution the situation, however, is different. Once a splitting is generated, e.g. using a veto algorithm [53, 98], we need to re-compute $x \rightarrow x / z$ because each emitted parton increases the combined $x$ value. The evolution then proceeds with this different effective splitting kernel. In other words, the PDF dependence in eq. (3.11) explicitly correlates parton emissions, breaking a key ingredient to the Poisson derivation.

To quantify this effect we numerically evaluate the gluon emission spectrum generated by the Sudakov form factor given in eq. (3.11) using a veto algorithm. We neglect any recoil 
effects and therefore expect a somewhat smaller suppression in the first bin. In figure 6 we display the normalized ratios of the gluon production rates off initial state $d$-quarks. We assume different starting values for $x^{(0)}$ thereby keeping the evolution distance and the minimum step size of $\Delta x=0.02$ fixed. This roughly corresponds to the emission of a hard additional jet with $p_{T} \approx 70 \mathrm{GeV}$ which we are interested in for the LHC. The starting scale we vary between $x^{(0)}=0.03$ and $x^{(0)}=0.2$ corresponding to a hard process of $105 \mathrm{GeV}$ and $700 \mathrm{GeV}$ respectively. The jet ratios strongly deviate from the Poisson pattern for low $x^{(0)}$, while the effect quickly diminishes for large $x^{(0)}$. This is the same pattern we find for the PDF effect in section 3.2.

Finally, to see how our two approaches to parton density effects are related we study the PDF part of the weight attached to a single resolvable emission coming from a collision with momentum fraction $x_{0}$. Using the backward evolution eq. (3.11) the exclusive one-jet rate can be represented as

$$
\begin{aligned}
\sigma_{1} & \sim f_{1}\left(x^{(0)}, Q^{2}\right) f_{2}\left(x^{(0)}, Q^{2}\right) \sigma_{0}^{\text {partonic }} \int \frac{d t}{t} \int \frac{d z}{z} P(z) \frac{f\left(x^{(0)} / z, t\right)}{f\left(x^{(0)}, t\right)} \\
& \sim f_{1}\left(x^{(0)}, Q^{2}\right) f_{2}\left(x^{(0)}, Q^{2}\right) \sigma_{0}^{\text {partonic }} \frac{f\left(x^{(1)}, Q^{2}\right)}{f\left(x^{(0)}, Q^{2}\right)} \frac{P\left(z^{(1)}\right)}{z^{(1)}} .
\end{aligned}
$$

In the second line we limit ourselves to the leading logarithmic approximation (i.e. ignoring the $t$ dependence of the PDFs) and fix the resolvable momentum fraction to its threshold value $z^{(1)}=x^{(0)} / x^{(1)}$. The effect of the PDF weight in the ratio $\sigma_{1} / \sigma_{0}$ then turns into a suppression factor $f\left(x^{(1)}, Q^{2}\right) / f\left(x^{(0)}, Q^{2}\right)$. Each emission just pushes up the overall PDF suppression and we are effectively led to the estimate on the shape of the ratios provided by eq. (3.9).

\subsection{BFKL evolution}

So far, we only consider the DGLAP evolution which relies on collinear factorization and resums collinear logarithms. Parton evolution can also be represented by BFKL [99, 100] or CCFM [101, 102] dynamics which rely on an entirely different form of the factorized matrix element. In this approach a simple expression for the $n$-jet generating function at leading $\operatorname{logarithmic}$ order in $\log 1 / x$ and $\log Q / p_{V}$ reads [103]

$$
\Phi\left(Q^{2}, p_{V}^{2}\right)_{\mathrm{BFKL}}=\exp \left(-\frac{2 C_{A} \alpha_{s}}{\pi w} \log \frac{Q}{p_{V}}\right)\left[1+(1-u) \frac{2 C_{A} \alpha_{s}}{\pi w} \log \frac{Q}{p_{V}}\right]^{u /(1-u)}
$$

In this expression $w$ is the Mellin conjugate variable of $x$ which for the physical jet rates requires convolution with the structure functions and transformation back to $x$ space. In analogy to eq. (2.9), functional derivatives of $\Phi_{\mathrm{BFKL}}$ evaluated at $u=0$ return exclusive jet rates.

As in the DGLAP case of section 2.1 we can compute the scaling patterns in the limit of small and large emission probability. 
(1) $\frac{2 C_{A} \alpha_{s}}{\pi w} \log \frac{Q}{p_{V}} \gg 1$

Following eq. (3.13) this large logarithm describes the limit of large emission probabilities. Again, we find that the $n_{\text {jets }}$ distribution shows a Poisson scaling

$$
\left.\frac{1}{n !} \frac{\partial^{n}}{\partial u^{n}}\left[1+(1-u) \frac{2 C_{A} \alpha_{s}}{\pi w} \log \frac{Q}{p_{V}}\right]^{u /(1-u)}\right|_{u=0} \approx \frac{1}{n !} \log ^{n}\left(1+\frac{2 C_{A} \alpha_{s}}{\pi w} \log \frac{Q}{p_{V}}\right) .
$$

Note that this result is obtained by taking the limit after the differentiation. Although eq. (3.14) is formally true in the limit $1 / w \log Q / p_{V} \rightarrow \infty$ the enhancement of the Poisson term is gradual, i.e. $\log ^{n}\left(1 / w \log Q / p_{V}\right)$. At every multiplicity there appear terms of the order $\log ^{n-1}\left(1 / w \log Q / p_{V}\right)$ with possibly large coefficients. Therefore, we expect this Poisson distribution to only emerge at very high energies and currently experimentally inaccessible $x$ values.

(2) $\frac{2 C_{A} \alpha_{s}}{\pi w} \log \frac{Q}{p_{V}} \ll 1$

Expanding to leading order in the emission probability the rates are

$$
P_{n} \approx\left(\frac{2 C_{A} \alpha_{s}}{\pi w} \log \frac{Q}{p_{V}}\right)^{n}
$$

This is a staircase distribution in the jet ratios. This result can also be seen in the corresponding fixed order computation [104]. Apparently, this staircase distribution is of an entirely different origin from the gluonic cascade in DGLAP, eq. (2.20). In the BFKL evolution there is no notion of subsequent emissions, all gluons are emitted directly from the factorized $t$-channel. A subsequent emission does not contain the $\log 1 / x$ enhancement. For the physical jet rates, we transform to $x$ space via a convolution and the rates are of course different; only the scaling, i.e. the shape of the ratio distribution, does not change.

Unlike the patterns we see in the DGLAP approach, both limits pointed to above are only realized in corners of phase space which are not particularly relevant to the hard processes at the LHC. The actual $n_{\text {jets }}$ distributions in BFKL-type regions will not be described in terms of a pure scaling pattern. In fact, it was pointed out in ref. [105] that the $n_{\text {jets }}$ distribution from simulated BFKL emissions are described better by a shifted Gaussian than a Poisson distribution. From a scaling perspective it is difficult to imagine how this might be induced by first principles QCD.

\section{LHC predictions}

ATLAS and CMS analyses based on 2011 and 2012 data with an excellent understanding of the detectors allows for detailed investigations of high-jet-multiplicity final states. The scaling patterns derived in this work should hence be experimentally testable before the 2013 shutdown. 
From the discussion in section 3 we know that $n$-jet scaling arguments can be transferred from $e^{+} e^{-}$to hadron colliders without major modifications. This means we can search for Poisson scaling and staircase scaling in standard candle processes at the LHC. For reasonably large jet multiplicities both of these patterns are derived from first principles QCD. Parton density effects modify the first few bins in the exclusive jet ratios $R_{(n+1) / n}$.

In the definition of the observables we need to carefully distinguish between inclusive and exclusive jet rates, i.e. counting exactly $n$ jets or at least $n$ jets in the $n$-jet bin radiated off a given hard processes. Staircase scaling is unique in the sense that it appears in exclusive and inclusive observables with the same constant ratio $R_{(n+1) / n}$ [1]. Poisson scaling at $e^{+} e^{-}$colliders or hadron colliders is limited to exclusive jet rates. Its translation into inclusive observables is tedious [1].

Following section 3 we expect that QCD features together with the PDF effects produce a convincing staircase pattern for cross sections with a democratic jet selection and in the absence of major kinematic cuts $[1,13]$. The ratios $R_{(n+1) / n}$ should be constant, eq. (1.2). Towards large jet multiplicities this constant value $R$ is largely independent of the underlying hard process. The first $n_{\text {jets }}$ bin is most sensitive to the parton densities, as shown in section 3.2. The PDF effect will generally lead to a suppression of the otherwise large value of $R_{1 / 0}$, extending the staircase pattern to low multiplicities where the QCD derivation fails. However, the PDF effect is clearly not independent of the hard core process which can involve incoming gluons as well as incoming quarks.

In contrast, for a jet selection with a large ratio between the core-process scale and the jet acceptance cut $\left(p_{T, j} \geq p_{V}\right)$ we expect several bins of the $n_{\text {jets }}$ distribution to follow a Poisson distribution. Only at large jet multiplicities the ratios will overshoot the Poisson pattern $\bar{n} /(n+1)$, turning into a staircase tail. This is the same behavior we derive for $e^{+} e^{-} \rightarrow$ jets production in section 2 .

Our considerations about the $n_{\text {jets }}$ distributions associated with a general hard process needs to be validated for a variety of channels, potentially in dedicated analyses. As a starting point, we propose two simple extensions of already existing analyses. They serve as stringent tests in particular of the Poisson hypothesis for the low-multiplicity bins and the transition to a staircase-like behavior for higher $n_{\text {jets }}$ bins. We start with the Drell-Yan process, but asking for a leading jet with significant transverse momentum $p_{T}^{\text {lead }} \geq 5 p_{V}$ while all additional jets are selected democratically with $p_{T} \geq p_{V}$. Our second example is the $n_{\text {jets }}$ distribution inside a rapidity interval defined by a dijet core system.

\section{1 $\mathrm{Z}+$ jets}

For the $W / Z+$ jets channels recent LHC measurements of the $n_{\text {jets }}$ distribution support a staircase hypothesis $[73-79,94]$. The key ingredient is the democratic selection of jets, e.g. $p_{T, j}>30 \mathrm{GeV}$ for all jets in $Z \rightarrow \mu^{+} \mu^{-}$and $Z \rightarrow e^{+} e^{-}$events [94]. We propose a minimal modification of this analysis by increasing the $p_{T}$ cut on the leading jet to $p_{T}^{\text {lead }}>150 \mathrm{GeV}$. The hard core process is now defined as the $Z$ boson combined with the leading jet, the first of $n_{\text {jets }}$ radiated QCD jets is the second jet in the event. According to section 3.2 such a selection makes the PDF suppression effect rather marginal and we expect to see a Poisson distribution in the low-multiplicity bins. 

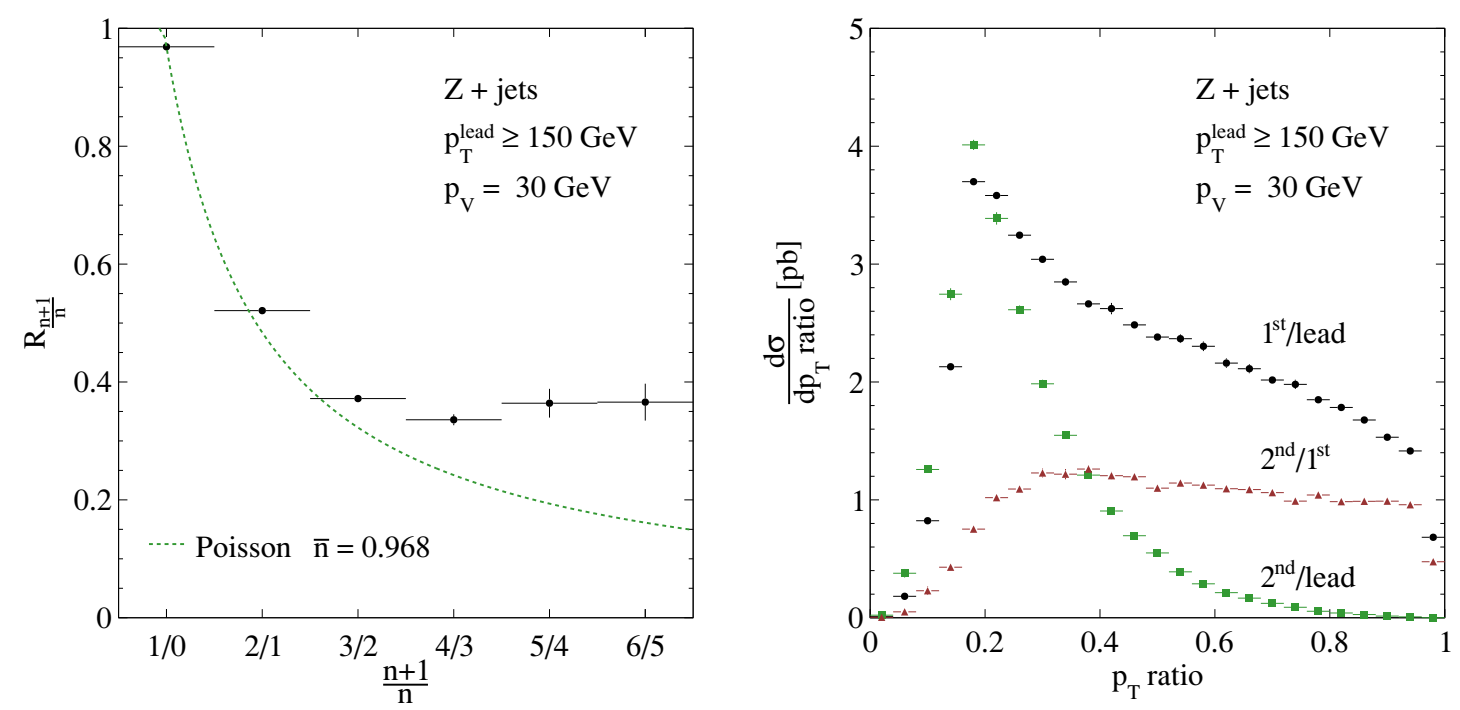

Figure 7. Left panel: exclusive jet ratios for $Z+$ jets production at $\sqrt{S}=7 \mathrm{TeV}$. We require a leading jet with $p_{T}^{\text {lead }} \geq 150 \mathrm{GeV}$. All other jets have $p_{T} \geq p_{V}=30 \mathrm{GeV}$. The line shows a Poisson shape with $\bar{n}$ extracted from the first bin. Right panel: cross sections as a function of the event-wise ratios of the jet transverse momenta in inclusive $Z+3$ jet production. While the leading jet has to pass $p_{T}^{\text {lead }} \geq 150 \mathrm{GeV}$ additional jets are selected uniformly with just $p_{T} \geq p_{V}=30 \mathrm{GeV}$.

To simulate this process we use Sherpa v1.4.0 [57, 58, 90]. We employ Sherpa's treelevel merging algorithm based on truncated showers [106, 107], including real-emission matrix elements for up to five final state partons, the merging scale we set to $25 \mathrm{GeV}$. In figure 7 we present the exclusive $n_{\text {jets }}$ cross section ratios, keeping in mind that the counting of the radiated jets does not include the hard leading jet which together with the $Z$ boson constitutes the core process. As expected, we observe a clear Poisson scaling for $n_{\text {jets }} \leq 3$. This can be seen when comparing the individual bins to the Poisson shape $R_{(n+1) / n}=\bar{n} /(n+1)$ where $\bar{n}$ is fixed by the first bin $R_{1 / 0}$.

However, already the three-jet rate comes out higher than the Poisson extrapolation. At this point the non-abelian nature of QCD radiation takes over, giving us a staircase tail with $R \approx 0.36$. This behavior is strongly reminiscent of figure 2 for $e^{+} e^{-} \rightarrow$ jets. An updated analysis of the 2011 data sample and certainly the 2012 data set should allow for a test of up to five additional jets, probing QCD predictions for the complete $n_{\text {jets }}$ distribution.

In addition to its defining power of very general features of the $n_{\text {jets }}$ distribution the jet selection cuts are reflected in the transverse momentum distributions of the jets. In the right panel of figure 7 we depict the cross sections as a function of the ratios of the $p_{T}$-ordered jet transverse momenta defined event-by-event. For this distribution we for once deviate from our usual exclusive jet counting and consider events with at least three jets in the final state. We consider the $p_{T}$ ratios first-over-leading (black), second-overleading (green) and second-over-first jet (red). With the leading jet $p_{T} \geq 150 \mathrm{GeV}$ and the additional jets selected uniformly with $p_{T} \geq 30 \mathrm{GeV}$ we expect the first and second jet 



Figure 8. Left: predictions vs ATLAS data ([75] for additional plots see https://atlas .web.cern.ch/Atlas/GROUPS/PHYSICS/PAPERS/STDM-2011-03/) for the di-jet gap fraction $\left(p_{V}=20 \mathrm{GeV}\right)$ as a function of $\Delta y$ and in slices of $\bar{p}_{T}$. Right: average number of gap jets as a function of $\bar{p}_{T}$ in slices of $\Delta y$. The tagging jets are defined as most forward and most backward. All jets are reconstructed using the anti- $k_{t}$ algorithm with $R=0.6$.

to peak around the selection cut. This is confirmed by the simulated results that exhibit strong peaks for the corresponding ratios around $p_{T}^{\text {1st }} / p_{T}^{\text {lead }} \approx 0.2$ and $p_{T}^{2 \text { nd }} / p_{T}^{\text {lead }} \approx 0.2$. However, it is interesting to note that the ratio $p_{T}^{2 \text { nd }} / p_{T}^{1 \text { st }}$ does not peak around 1 . Rather QCD favors the first radiated jet to be significantly harder than the second. The obtained distribution in fact turns out to be more or less flat between 0.25 and 1 . It is certainly interesting to study these observables in addition to the $n_{\text {jets }}$ distribution, as they contain complementary information on the underlying QCD dynamics.

\subsection{QCD gap jets}

An interesting set of observables from the perspective of multi-jet final states are gap fractions or gap jets. In that case we require a specific kinematic structure of hard and widely separated jets and count the QCD jets in between. The core process is the production of two widely separated hard jets.

A recent ATLAS study [75] identifies two forward jets, so-called tagging jets, either as the highest $p_{T}$ (selection $\mathrm{A}$ ) or the most forward and backward in rapidity (selection $\mathrm{B}$ ). The core di-jet system is defined in terms of $\bar{p}_{T}=\left(p_{T, 1}+p_{T, 2}\right) / 2$ and $\Delta y=\left|y_{1}-y_{2}\right|$. The gap fraction $P_{0}=\sigma_{0} / \sigma_{\text {tot }}$ is given by all events with no additional jet in between the two tagging jets and $p_{T}>p_{V}=20 \mathrm{GeV}$. ATLAS measures it as a function of $\Delta y$. In addition, they provide us with the average multiplicity of vetoed jets or gap jets. Following section 2 

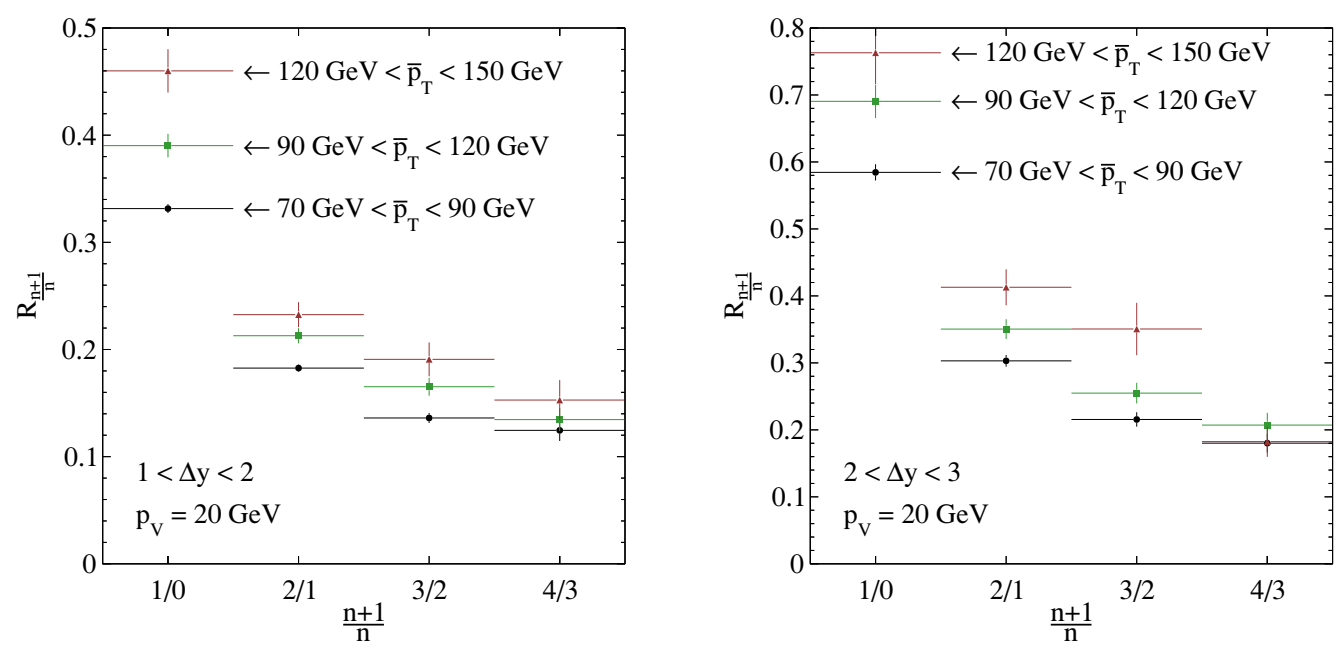

Figure 9. Jet ratios for exclusive gap jets in slices of $\bar{p}_{T}$ for the tagging jets, defined as the most forward and backward in the event. The rapidity separation of the gap-defining jets is $1<\Delta y<2$ (left) and $2<\Delta y<3$ (right).

a hard cut on $\bar{p}_{T}$ will typically enforce a larger logarithm than simply $\log \left(\bar{p}_{T} / p_{V}\right)$. This is because the two tagging jets tend to be asymmetric in $p_{T}$. As a consequence we expect a Poisson scaling for the gap jets.

Perturbatively, the gap fractions are dominated by the single emission probability while the average number of gap jets probes multiple emissions. We again propose a simple extension of the existing analysis. In addition to the average number of gap jets the corresponding exclusive $n_{\text {jets }}$ distribution should be studied. Reproducing the average number of gap jets cannot validate a full $n_{\text {jets }}$ distribution, where the latter directly tests QCD scaling patterns.

Again, we use Sherpa with tree-level merging, including hadronization and underlying event. We account for up to four hard final state partons in the veto region, the merging scale set to $Q_{\text {cut }}=20 \mathrm{GeV}$. This way, the emission of wide-angle soft gluons should be correctly modeled including the full color structure. The DGLAP based parton shower resums logarithms of large $p_{T}$ ratios only, for sufficiently large rapidity gaps one may expect the agreement to suffer $[114,115]$. In figure 8 we compare Sherpa predictions with ATLAS data [75] for both the gap fraction and the average number of jets in selection B. For both observables the agreement with data is excellent. Even for large $\Delta y>4$ the simulation works well within the statistical limitations, so we do not see the merging of tree-level matrix elements with the parton shower breaking down. A detailed analysis of the same data using the MC@NLO implementation in Sherpa and assessing both perturbative and non-perturbative theoretical uncertainties is presented in ref. [116].

With the confidence gained by correctly modeling the gap fraction and the average number of gap jets, we proceed to predict the $n_{\text {jets }}$ distribution for gap jets. In the forwardbackward selection this includes all resolved jets with $p_{T}>p_{V}$. In figure 9 we present the gap jet multiplicity ratios for two different rapidity separations, $1<\Delta y<2$ (left) and 
$2<\Delta y<3$ (right), and in slices of $\bar{p}_{T}$. Enforcing a large ratio between the $p_{T}$ of the tagging jets versus the jet-counting scale $p_{V}=20 \mathrm{GeV}$ induces a Poisson distribution for all the considered $\bar{p}_{T}$ selections. Comparing the two panels of figure 9 we see that the shape, though not the normalization, of the $n_{\text {jets }}$ distributions is largely independent of $\Delta y$. For large $\bar{p}_{T}$ the highest jet multiplicities suffer from non-negligible statistical fluctuations, in particular for the $4 / 3$-jet bin.

For kinematical selections which induce a Poisson scaling we can approximately derive the average number of gap jets from $R_{1 / 0}$. This number determines $\bar{n}$ and allows for a straightforward calculation of the veto survival probability or gap fraction, $P_{0}=\exp (-\bar{n})$. Comparing this prediction to the explicit $\bar{n}$ measurement of in figure 8 we find both values consistent. Such a comparison will provide insight into jet-veto survival probabilities, not only for the analysis presented here but also in the context of Higgs analyses [1, 117-123].

\section{Conclusions}

While it has been known for a long time that the exclusive $n_{\text {jets }}$ distribution at hadron colliders follows simple scaling features [10-12], a proper understanding starting from first principles QCD has been missing. The two underlying scaling patterns for jet emission is a Poisson shape or staircase scaling.

1. Poisson scaling is well known from multi-jet final states in $e^{+} e^{-}$production. It can be easily derived in the abelian limit of QCD, i.e. gluon radiation off hard quark legs, in complete analogy to soft photon emission in QED. Using the parton shower picture as well as generating functionals we have found that a Poisson distribution is tied to a large hierarchy of scales and a logarithmically enhanced radiation matrix element. Such different scales can be induced by kinematic cuts, for example in $Z+$ jets [1] or $\gamma+$ jets [13] production. Many effects, like non-abelian gluon splittings, phase space limitations, or sub-leading logarithms modify the pure Poisson shape of the exclusive $n_{\text {jets }}$ distribution.

2. Staircase scaling [10-12] is defined as constant exclusive jet ratios $R_{(n+1) / n}=$ $\sigma_{n+1} / \sigma_{n}=R$. It has been observed at hadron colliders since UA1 and generally appears for a democratic jet selection, avoiding large scale separations. This includes Higgs production in gluon fusion and in weak boson fusion [1]. For large jet multiplicities we have found that staircase scaling can be predicted as a non-abelian QCD effect. At $e^{+} e^{-}$colliders as well as at hadron colliders it describes the exclusive $n_{\text {jets }}$ distribution for large jet multiplicities. At lower jet multiplicities parton density effects violate our picture of jet radiation off an unchanged hard process. Numerically, this correction restores the staircase pattern, often down to the first emitted jet.

After this derivation and study of the two generic patterns which are based only on QCD and patterns of the parton densities this prediction should be tested experimentally. We present two minor modifications of current LHC analyses which would be well suited to probe the universal nature of our findings. First, the classical $Z+$ jets channel should 
be used to study the transition from Poisson to staircase scaling using a simple kinematic cut on the leading jet. A similar proposal exists for $\gamma+$ jets production [13]. In addition, QCD gap jets already probe these scaling features through the measured average number of gap jets. Extending the main observable from the mean number of gap jets to the $n_{\text {jets }}$ distributions would allow us to confirm what our first estimate suggests - that gap jets can be described precisely using the transition from staircase to Poisson scaling.

While from a perturbative QCD point of view the exclusive number of jets $n_{\text {jets }}$ is not a particularly attractive observable, it clearly has huge benefits in LHC analyses. In the future, jet vetoes in Higgs searches [1] are going to become more and more relevant, separating the different underlying production processes. In this paper we have shown that it is possible to predict the main features of the $n_{\text {jets }}$ distribution from QCD. Testing the universality of these features would give us a new and improved tool for a huge number of LHC analyses to come.

\section{Acknowledgments}

We would like to thank Bryan Webber, Einan Gardi, Jennifer Smilie, Patrick Meade and Peter Zerwas for enlightening discussions as well as the Göttingen ATLAS group for experimental input. Moreover, we are grateful to Michelangelo Mangano for setting this problem. EG and SS acknowledge financial support by the Helmholtz Alliance 'Physics at the Terascale' and BMBF under contract 05H12MG5. PS acknowledges support by the IMPRS for Precision Tests of Fundamental Symmetries.

\section{A Iterated Poisson process}

The process generated by summing an arbitrary number of independent Poisson processes $P=P\left(\bar{n}_{1}\right)+P\left(\bar{n}_{2}\right)+\cdots P\left(\bar{n}_{n}\right)$ is itself a Poisson process with the expectation value $\bar{n}=\bar{n}_{1}+\bar{n}_{1}+\cdots \bar{n}_{n}$. In order to deviate from a Poisson shape, we allow for the occurrence of subsequent splittings. A QCD example would be gluon radiation off a quark line with a subsequent splitting of the gluon into two gluons or two quarks.

Suppose we start with a single mother Poisson process described by the parameter $\bar{n}$. Every emission by the mother process generates a daughter process which itself evolves through the same scale as an independent Poisson process with expectation $\bar{n}^{\prime}$. In general we will have $\bar{n} \neq \bar{n}^{\prime}$. For the mother (daughter) process with Poisson parameter $\bar{n}\left(\bar{n}^{\prime}\right)$, the two parameter distribution can be approximated by

$$
\begin{aligned}
P\left(n ; \bar{n}, \bar{n}^{\prime}\right) & =e^{-\bar{n}-n \bar{n}^{\prime}} \sum_{i=0}^{n}\left(\frac{(n-1) !}{i !(n-i-1) !(n-i) !}\right) \bar{n}^{\prime i} \bar{n}^{n-i} \\
& =\frac{e^{-\bar{n}-n \bar{n}^{\prime}} \bar{n}^{n} \bar{n}^{\prime n-1}}{n !}\left|\operatorname{HG}\left[1-n, 2,-\bar{n} / \bar{n}^{\prime}\right]\right|
\end{aligned}
$$

where HG is the confluent hypergeometric function with integral representation

$$
\mathrm{HG}[a, b, z]=\frac{1}{\Gamma(a)} \int_{0}^{\infty} e^{-z t} t^{a-1}(1+t)^{b-a-1} d t .
$$


Let us note some observations regarding the physics content of this model:

1. The exponential Poisson model is reproduced in the limit $\bar{n}^{\prime} \rightarrow 0$, where the additional splitting probability vanishes.

2. The zero emission probability is unchanged, while the one emission rate includes the additional non-splitting probability of the daughter process. It contains an additional suppression $e^{-\bar{n}^{\prime}}$ which universally reduces the first $n$-jet ratio for the iterated Poisson process.

3. In the limit of a large additional splitting probability $\bar{n}^{\prime} \gg \bar{n}$ the theory displays staircase scaling. The highest order term in eq. (A.1) carries a compensating factor of $n$ ! in the numerator.

4. The convergence of eq. (A.1) is guaranteed for all $\bar{n}$ and $\bar{n}^{\prime}$. However, inclusive unitarity of the cross-section is not possible; the sum over $n$ in eq. (A.1) is smaller than one for all $\bar{n}^{\prime} \neq 0$. We expect then that our toy model undershoots the full calculation, as confirmed by the $e^{+} e^{-}$jet fractions (see figure 11).

5. We emphasize that the purely probabilistic model given by eq. (A.1) does not capture all effects of leading-log QCD. In order to promote our toy model to full double leading logarithmically accurate QCD as far as the jet rates are concerned, we need to make two modifications. First of all we need to include the full Poisson history by hand (i.e. no recursive formula is achievable), and second, we require $\bar{n}^{\prime}$ to change as a function of the "generation" of subsequent emissions. More precisely the fully nonabelian $\sim C_{F} C_{A}^{2}$ three-gluon rate is correctly described if $\bar{n}^{\prime \prime}=(6 / 5) \overline{n^{\prime}}$. This value for $\bar{n}^{\prime \prime}$ then correctly describes the $C_{F}^{2} C_{A}^{2}$ coefficient to the four-gluon rate. In this manner we can essentially boot-strap the leading coefficients.

The important consequence of eq. (A.1) for realistic values of $\bar{n}^{\prime}>0$ is that the jet ratios $R_{(n+1) / n}$ are flatter than the naive Poisson expectation. In figure 10, we display the jet ratios generated by eq. (A.1) for $\bar{n}=\bar{n}^{\prime}$. By making analytic estimates for the subsequent splitting parameter we can compare our toy model with jet rates predictions from QCD.

We assume the relation between the two expectation values $\bar{n}$ and $\bar{n}^{\prime}$ to scale with the relative splitting probability for the simplest non-abelian emission. The 2-jet fraction at $\mathcal{O}\left(a^{2}\right)$ in double logarithmic approximation then gives $\bar{n} / \bar{n}^{\prime}=12 C_{F} / C_{A}$. For $\bar{n}<1$, the specific value of the two Poisson parameters do not affect the shape as long as their ratio is held fixed. Therefore, we compare the shapes of the iterated Poisson model with the jet fractions by normalizing the first ratio. This also allows a simple comparison to a purely Poisson process with ratios $1 /(n+1)$. The results are shown in figure 11 , where we find that the $P_{2} / P_{1}$ ratios are in exact agreement. Using eq. (A.1) the ratio is simply $\bar{n} / 2+\bar{n}^{\prime}$, which our choice of $\bar{n}^{\prime}$ reproduces precisely. The higher bins slightly undershoot the jet fractions in the iterated Poisson model. The reason stated previously is that the $\bar{n}^{\prime}$ should change as a function of the "generations" and the certain contribution need to be added by hand. The first evidence of this is contained at $\mathcal{O}\left(a^{3}\right)$ in $P_{3}$. The jet fraction coefficients 


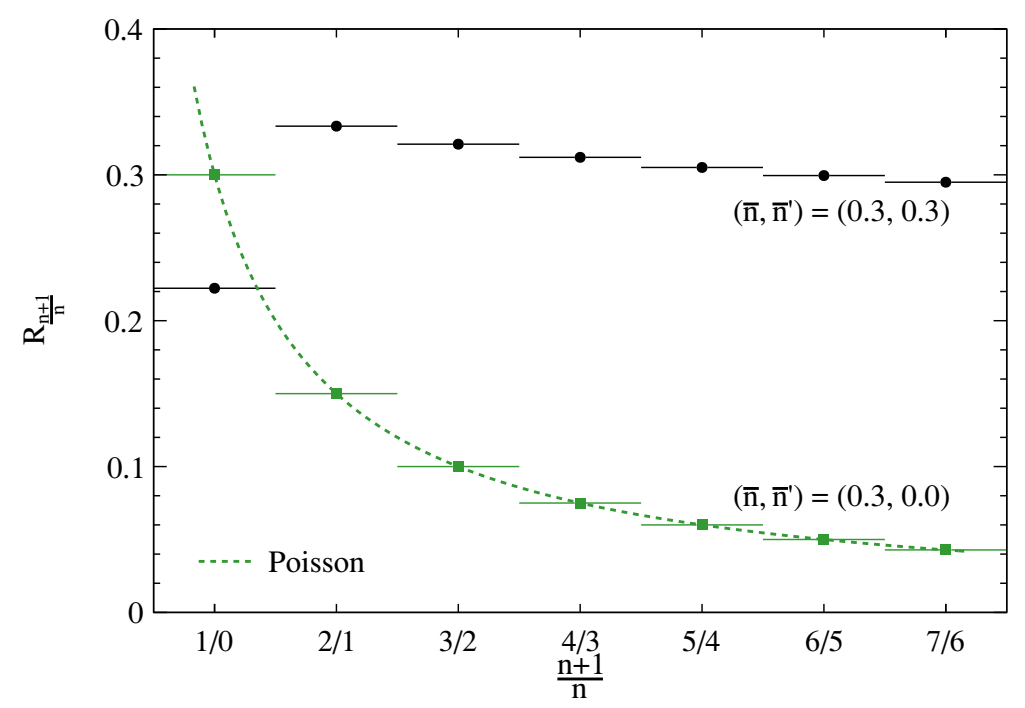

Figure 10. Ratios from an iterated (normal) Poisson process for $\bar{n}=0.3$ and $\bar{n}^{\prime}=3\left(\bar{n}^{\prime}=0\right)$ given in eq. (A.1). In the iterated case we see a general flattening of the distribution.

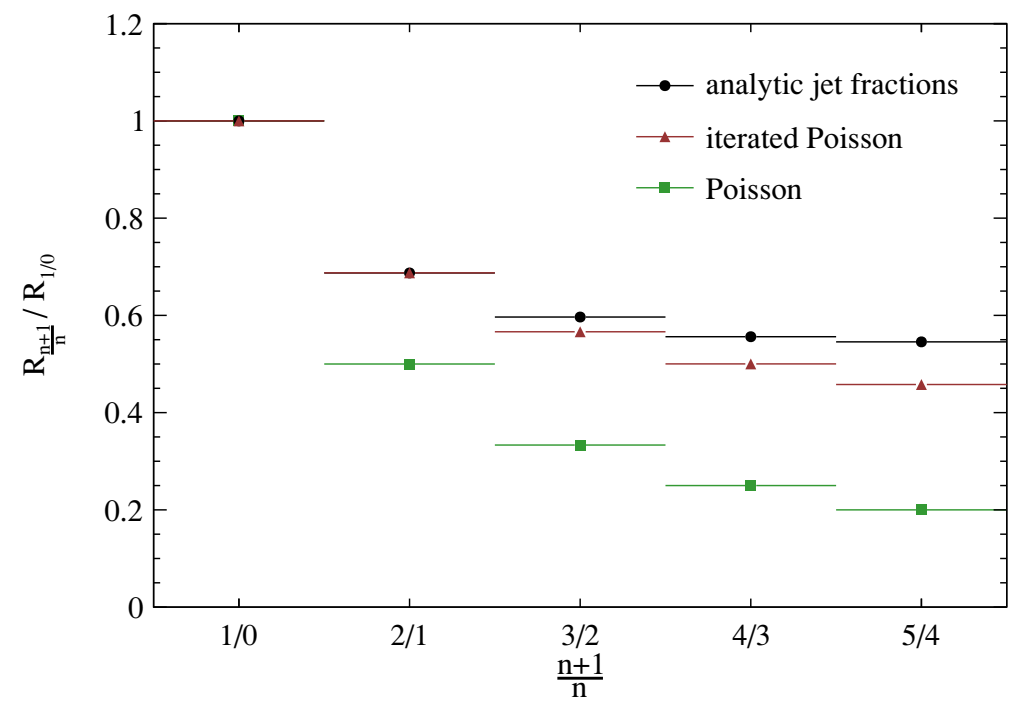

Figure 11. Ratios of $e^{+} e^{-} \rightarrow q \bar{q}+\mathrm{n} g$ jet fractions (black) as computed in appendix B compared to the iterated Poisson model with $\bar{n}^{\prime}=12 C_{F} / C_{A} \bar{n}$ (red), and Poisson scaling (green).

for the $\bar{n} \bar{n}^{\prime}$ and $\bar{n}^{\prime 2}$ terms are $1 / 12$ and 1/90 respectively, while the iterated Poisson model gives $1 / 12$ and $1 / 144$. For higher terms still the discrepancy persists. The conclusion from this plot is that the purely final state gluon cascade generates a staircase scaling pattern at higher multiplicities but seemingly fails to explain the suppression on the lower multiplicity bins. For this we need to account for PDFs. 


\section{B Jet fractions}

We briefly quote $e^{+} e^{-} \rightarrow q \bar{q}+n g$ jet fractions at $\mathcal{O}\left(\alpha_{s}^{5} L^{10}\right)$ in double logarithmic approximation and for the Durham algorithm. These are used to compare the relative size of subsequent terms in a purely final state cascade picture where the single emission probability is still smaller than unity. Results can be obtained from refs. [89, 124-126]. The abelian contributions are shown here to all orders such that in the limit $C_{A} \rightarrow 0$ we recover Poisson scaling. Defining $a=\alpha_{s} / \pi, L=\log y_{\text {cut }}$ and $P_{i}=\sigma_{i} / \sigma_{\text {tot }}$ we have:

$$
\begin{aligned}
& P_{0}=\exp \left[-\frac{a C_{F} L^{2}}{2}\right] \text {, } \\
& P_{1}=\left(\frac{a C_{F} L^{2}}{2}\right) \exp \left[-\frac{a C_{F} L^{2}}{2}\right]-a^{2}\left(\frac{C_{F} C_{A}}{48}\right) L^{4}+a^{3}\left(\frac{C_{F}^{2} C_{A}}{96}+\frac{C_{F} C_{A}^{2}}{960}\right) L^{6} \\
& -a^{4}\left(\frac{C_{F}^{3} C_{A}}{384}+\frac{C_{F}^{2} C_{A}^{2}}{1920}+\frac{C_{F} C_{A}^{3}}{21504}\right) L^{8} \\
& +a^{5}\left(\frac{C_{F}^{4} C_{A}}{2304}+\frac{C_{F}^{3} C_{A}^{2}}{7680}+\frac{C_{F}^{2} C_{A}^{3}}{43008}+\frac{C_{F} C_{A}^{4}}{552960}\right) L^{10}, \\
& P_{2}=\frac{1}{2 !}\left(\frac{a C_{F} L^{2}}{2}\right)^{2} \exp \left[-\frac{a C_{F} L^{2}}{2}\right]+a^{2}\left(\frac{C_{F} C_{A}}{48}\right) L^{4}-a^{3}\left(\frac{C_{F}^{2} C_{A}}{48}+\frac{7 C_{F} C_{A}^{2}}{2880}\right) L^{6} \\
& +a^{4}\left(\frac{C_{F}^{3} C_{A}}{128}+\frac{C_{F}^{2} C_{A}^{2}}{512}+\frac{C_{F} C_{A}^{3}}{5120}\right) L^{8} \\
& -a^{5}\left(\frac{C_{F}^{4} C_{A}}{576}+\frac{31 C_{F}^{3} C_{A}^{2}}{46080}+\frac{23 C_{F}^{2} C_{A}^{3}}{161280}+\frac{C_{F} C_{A}^{4}}{806400}\right) L^{10}, \\
& P_{3}=\frac{1}{3 !}\left(\frac{a C_{F} L^{2}}{2}\right)^{3} \exp \left[-\frac{a C_{F} L^{2}}{2}\right]+a^{3}\left(\frac{C_{F}^{2} C_{A}}{96}+\frac{C_{F} C_{A}^{2}}{720}\right) L^{6} \\
& -a^{4}\left(\frac{C_{F}^{3} C_{A}}{128}+\frac{3 C_{F}^{2} C_{A}^{2}}{1280}+\frac{41 C_{F} C_{A}^{3}}{161280}\right) L^{8} \\
& +a^{5}\left(\frac{C_{F}^{4} C_{A}}{384}+\frac{19 C_{F}^{3} C_{A}^{2}}{15360}+\frac{115 C_{F}^{2} C_{A}^{3}}{387072}+\frac{13 C_{F} C_{A}^{4}}{460800}\right) L^{10}, \\
& P_{4}=\frac{1}{4 !}\left(\frac{a C_{F} L^{2}}{2}\right)^{4} \exp \left[-\frac{a C_{F} L^{2}}{2}\right]+a^{4}\left(\frac{C_{F}^{3} C_{A}}{384}+\frac{7 C_{F}^{2} C_{A}^{2}}{7680}+\frac{17 C_{F} C_{A}^{3}}{161280}\right) L^{8} \\
& -a^{5}\left(\frac{C_{F}^{4} C_{A}}{576}+\frac{19 C_{F}^{3} C_{A}^{2}}{1024}+\frac{251 C_{F}^{2} C_{A}^{3}}{967680}+\frac{151 C_{F} C_{A}^{4}}{5806080}\right) L^{10}, \\
& P_{5}=\frac{1}{5 !}\left(\frac{a C_{F} L^{2}}{2}\right)^{5} \exp \left[-\frac{a C_{F} L^{2}}{2}\right] \\
& +a^{5}\left(\frac{C_{F}^{4} C_{A}}{2304}+\frac{13 C_{F}^{3} C_{A}^{2}}{46080}+\frac{79 C_{F}^{2} C_{A}^{3}}{967680}+\frac{31 C_{F} C_{A}^{4}}{3628800}\right) L^{10}, \\
& P_{n \geq 6}=\frac{1}{n !}\left(\frac{a C_{F} L^{2}}{2}\right)^{n} \exp \left[-\frac{a C_{F} L^{2}}{2}\right] \text {. }
\end{aligned}
$$

Open Access. This article is distributed under the terms of the Creative Commons Attribution License which permits any use, distribution and reproduction in any medium, provided the original author(s) and source are credited. 


\section{References}

[1] E. Gerwick, T. Plehn and S. Schumann, Understanding jet scaling and jet vetos in Higgs searches, Phys. Rev. Lett. 108 (2012) 032003 [arXiv:1108.3335] [InSPIRE].

[2] ATLAS collaboration, G. Aad et al., Measurement of $t \bar{t}$ production with a veto on additional central jet activity in pp collisions at $\sqrt{s}=7$ TeV using the ATLAS detector, Eur. Phys. J. C 72 (2012) 2043 [arXiv:1203.5015] [INSPIRE].

[3] ATLAS collaboration, G. Aad et al., Measurements of top quark pair relative differential cross-sections with ATLAS in pp collisions at $\sqrt{s}=7$ TeV, arXiv:1207.5644 [INSPIRE].

[4] F. Kling, T. Plehn and M. Takeuchi, Tagging single tops, arXiv: 1207.4787 [INSPIRE].

[5] C. Englert, T. Plehn, P. Schichtel and S. Schumann, Jets plus missing energy with an autofocus, Phys. Rev. D 83 (2011) 095009 [arXiv:1102.4615] [INSPIRE].

[6] T. Plehn and T.M. Tait, Seeking sgluons, J. Phys. G 36 (2009) 075001 [arXiv:0810.3919] [INSPIRE].

[7] S. Schumann, A. Renaud and D. Zerwas, Hadronically decaying color-adjoint scalars at the LHC, JHEP 09 (2011) 074 [arXiv: 1108.2957] [INSPIRE].

[8] H.K. Dreiner, M. Krämer and J. Tattersall, How low can SUSY go? matching, monojets and compressed spectra, Europhys. Lett. 99 (2012) 61001 [arXiv:1207.1613] [INSPIRE].

[9] D.E. Morrissey, T. Plehn and T.M. Tait, Physics searches at the LHC, Phys. Rept. 515 (2012) 1 [arXiv:0912.3259] [INSPIRE].

[10] S. Ellis, R. Kleiss and W.J. Stirling, W's, Z's and jets, Phys. Lett. B 154 (1985) 435 [INSPIRE].

[11] F.A. Berends, W. Giele, H. Kuijf, R. Kleiss and W.J. Stirling, Multi-jet production in $W, Z$



[12] F.A. Berends, H. Kuijf, B. Tausk and W. Giele, On the production of a $W$ and jets at hadron colliders, Nucl. Phys. B 357 (1991) 32 [InSPIRE].

[13] C. Englert, T. Plehn, P. Schichtel and S. Schumann, Establishing jet scaling patterns with a photon, JHEP 02 (2012) 030 [arXiv:1108.5473] [INSPIRE].

[14] Y.L. Dokshitzer, V.A. Khoze, A.H. Mueller and S.I. Troian, Basics of perturbative QCD, Ed. Frontieres, Gif-sur-Yvette France (1991).

[15] R.K. Ellis, W.J. Stirling and B. Webber, QCD and collider physics, Camb. Monogr. Part. Phys. Nucl. Phys. Cosmol. 8 (1996) 1 [InSPIRE].

[16] S. Catani, F. Krauss, R. Kuhn and B. Webber, QCD matrix elements + parton showers, JHEP 11 (2001) 063 [hep-ph/0109231] [INSPIRE].

[17] F. Krauss, Matrix elements and parton showers in hadronic interactions, JHEP 08 (2002) 015 [hep-ph/0205283] [INSPIRE].

[18] M.L. Mangano, M. Moretti and R. Pittau, Multijet matrix elements and shower evolution in hadronic collisions: $W b \bar{b}+n$ jets as a case study, Nucl. Phys. B 632 (2002) 343 [hep-ph/0108069] [INSPIRE].

[19] T. Plehn, Lectures on LHC physics, Lect. Notes Phys. 844 (2012) 1 [arXiv:0910.4182] [INSPIRE]. 
[20] S. Catani, Y.L. Dokshitzer and B. Webber, The $K^{-}$perpendicular clustering algorithm for jets in deep inelastic scattering and hadron collisions, Phys. Lett. B 285 (1992) 291 [INSPIRE].

[21] S. Catani, B. Webber and Y. Dokshitzer, The $k_{T}$ clustering algorithm for jets in deep inelastic scattering, Nucl. Phys. Proc. Suppl. 29A (1992) 136 [InSPIRE].

[22] J. Alwall, M. Herquet, F. Maltoni, O. Mattelaer and T. Stelzer, MadGraph 5: going beyond, JHEP 06 (2011) 128 [arXiv:1106.0522] [INSPIRE].

[23] T. Gleisberg and S. Hoeche, Comix, a new matrix element generator, JHEP 12 (2008) 039 [arXiv:0808.3674] [INSPIRE].

[24] T. Becher and M. Neubert, Factorization and NNLL resummation for Higgs production with a jet veto, JHEP 07 (2012) 108 [arXiv:1205.3806] [INSPIRE].

[25] A. Banfi, G.P. Salam and G. Zanderighi, $N L L+N N L O$ predictions for jet-veto efficiencies in Higgs-boson and Drell-Yan production, JHEP 06 (2012) 159 [arXiv:1203.5773] [INSPIRE].

[26] Z. Bern et al., Four-jet production at the Large Hadron Collider at next-to-leading order in QCD, Phys. Rev. Lett. 109 (2012) 042001 [arXiv:1112.3940] [INSPIRE].

[27] C.F. Berger et al., Precise predictions for $W+4$ jet production at the Large Hadron Collider, Phys. Rev. Lett. 106 (2011) 092001 [arXiv: 1009.2338] [InSPIRE].

[28] H. Ita et al., Precise predictions for $Z+4$ jets at hadron colliders, Phys. Rev. D 85 (2012) 031501 [arXiv: 1108.2229] [INSPIRE].

[29] S. Dittmaier, P. Uwer and S. Weinzierl, NLO QCD corrections to $t \bar{t}+$ jet production at hadron colliders, Phys. Rev. Lett. 98 (2007) 262002 [hep-ph/0703120] [INSPIRE].

[30] A. Bredenstein, A. Denner, S. Dittmaier and S. Pozzorini, NLO QCD corrections to top anti-top bottom anti-bottom production at the LHC: 2. full hadronic results, JHEP 03 (2010) 021 [arXiv: 1001.4006] [INSPIRE].

[31] G. Bevilacqua, M. Czakon, C. Papadopoulos and M. Worek, Dominant QCD backgrounds in Higgs boson analyses at the LHC: a study of $p p \rightarrow t \bar{t}+2$ jets at next-to-leading order, Phys. Rev. Lett. 104 (2010) 162002 [arXiv:1002.4009] [InSPIRE].

[32] J.M. Campbell, R.K. Ellis and G. Zanderighi, Next-to-leading order Higgs +2 jet production via gluon fusion, JHEP 10 (2006) 028 [hep-ph/0608194] [INSPIRE].

[33] J.M. Campbell, R.K. Ellis and C. Williams, Hadronic production of a Higgs boson and two jets at next-to-leading order, Phys. Rev. D 81 (2010) 074023 [arXiv:1001.4495] [INSPIRE].

[34] G. Ossola, C.G. Papadopoulos and R. Pittau, Reducing full one-loop amplitudes to scalar integrals at the integrand level, Nucl. Phys. B 763 (2007) 147 [hep-ph/0609007] [InSPIRE].

[35] R.K. Ellis, W. Giele and Z. Kunszt, A numerical unitarity formalism for evaluating one-loop amplitudes, JHEP 03 (2008) 003 [arXiv:0708.2398] [INSPIRE].

[36] G. Ossola, C.G. Papadopoulos and R. Pittau, On the rational terms of the one-loop amplitudes, JHEP 05 (2008) 004 [arXiv:0802.1876] [INSPIRE].

[37] C.F. Berger et al., An automated implementation of on-shell methods for one-loop amplitudes, Phys. Rev. D 78 (2008) 036003 [arXiv:0803.4180] [INSPIRE].

[38] R.K. Ellis, W.T. Giele, Z. Kunszt and K. Melnikov, Masses, fermions and generalized D-dimensional unitarity, Nucl. Phys. B 822 (2009) 270 [arXiv:0806.3467] [INSPIRE]. 
[39] T. Binoth et al., A proposal for a standard interface between Monte Carlo tools and one-loop programs, Comput. Phys. Commun. 181 (2010) 1612 [arXiv:1001.1307] [INSPIRE].

[40] V. Hirschi et al., Automation of one-loop QCD corrections, JHEP 05 (2011) 044 [arXiv:1103.0621] [INSPIRE].

[41] G. Bevilacqua et al., HELAC-NLO, arXiv:1110.1499 [INSPIRE].

[42] F. Cascioli, P. Maierhofer and S. Pozzorini, Scattering amplitudes with open loops, Phys. Rev. Lett. 108 (2012) 111601 [arXiv:1111.5206] [INSPIRE].

[43] G. Cullen et al., Automated one-loop calculations with GoSam, Eur. Phys. J. C 72 (2012) 1889 [arXiv:1111.2034] [INSPIRE].

[44] T. Binoth et al., Automized squark-neutralino production to next-to-leading Order, Phys. Rev. D 84 (2011) 075005 [arXiv: 1108.1250] [InSPIRE].

[45] D. Goncalves-Netto, D. Lopez-Val, K. Mawatari, T. Plehn and I. Wigmore, Sgluon pair production to next-to-leading order, Phys. Rev. D 85 (2012) 114024 [arXiv:1203.6358] [INSPIRE].

[46] R. Boughezal, K. Melnikov and F. Petriello, A subtraction scheme for NNLO computations, Phys. Rev. D 85 (2012) 034025 [arXiv:1111.7041] [InSPIRE].

[47] T. Gehrmann and P.F. Monni, Antenna subtraction at NNLO with hadronic initial states: real-virtual initial-initial configurations, JHEP 12 (2011) 049 [arXiv:1107.4037] [INSPIRE].

[48] P. Baernreuther, M. Czakon and A. Mitov, Percent level precision physics at the Tevatron: first genuine NNLO QCD corrections to $q \bar{q} \rightarrow t \bar{t}+X$, Phys. Rev. Lett. 109 (2012) 132001 [arXiv: 1204.5201] [INSPIRE].

[49] R. Bonciani, S. Catani, M.L. Mangano and P. Nason, Sudakov resummation of multiparton QCD cross-sections, Phys. Lett. B 575 (2003) 268 [hep-ph/0307035] [INSPIRE].

[50] A. Banfi, G.P. Salam and G. Zanderighi, Principles of general final-state resummation and automated implementation, JHEP 03 (2005) 073 [hep-ph/0407286] [INSPIRE].

[51] A. Banfi, P.F. Monni, G.P. Salam and G. Zanderighi, Higgs and Z-boson production with a jet veto, arXiv:1206.4998 [INSPIRE].

[52] A. Buckley et al., General-purpose event generators for LHC physics, Phys. Rept. 504 (2011) 145 [arXiv:1101.2599] [InSPIRE].

[53] T. Sjöstrand, S. Mrenna and P.Z. Skands, PYTHIA 6.4 physics and manual, JHEP 05 (2006) 026 [hep-ph/0603175] [INSPIRE].

[54] T. Sjöstrand, S. Mrenna and P.Z. Skands, A brief introduction to PYTHIA 8.1, Comput. Phys. Commun. 178 (2008) 852 [arXiv:0710.3820] [INSPIRE].

[55] G. Corcella et al., HERWIG 6: an event generator for hadron emission reactions with interfering gluons (including supersymmetric processes), JHEP 01 (2001) 010 [hep-ph/0011363] [INSPIRE].

[56] M. Bahr et al., HERWIG++ physics and manual, Eur. Phys. J. C 58 (2008) 639 [arXiv: 0803.0883] [INSPIRE].

[57] T. Gleisberg et al., SHERPA 1.alpha: a proof of concept version, JHEP 02 (2004) 056 [hep-ph/0311263] [INSPIRE]. 
[58] T. Gleisberg et al., Event generation with SHERPA 1.1, JHEP 02 (2009) 007 [arXiv:0811.4622] [INSPIRE].

[59] L. Lönnblad, Correcting the color dipole cascade model with fixed order matrix elements, JHEP 05 (2002) 046 [hep-ph/0112284] [INSPIRE].

[60] L. Lönnblad and S. Prestel, Matching tree-level matrix elements with interleaved showers, JHEP 03 (2012) 019 [arXiv:1109.4829] [INSPIRE].

[61] J. Alwall et al., Comparative study of various algorithms for the merging of parton showers and matrix elements in hadronic collisions, Eur. Phys. J. C 53 (2008) 473 [arXiv:0706.2569] [INSPIRE].

[62] S. Frixione and B.R. Webber, Matching NLO QCD computations and parton shower simulations, JHEP 06 (2002) 029 [hep-ph/0204244] [INSPIRE].

[63] S. Hoeche, F. Krauss, M. Schonherr and F. Siegert, A critical appraisal of $N L O+P S$ matching methods, JHEP 09 (2012) 049 [arXiv:1111.1220] [INSPIRE].

[64] P. Nason, A new method for combining NLO QCD with shower Monte Carlo algorithms, JHEP 11 (2004) 040 [hep-ph/0409146] [INSPIRE].

[65] S. Frixione, P. Nason and C. Oleari, Matching NLO QCD computations with parton shower simulations: the POWHEG method, JHEP 11 (2007) 070 [arXiv: 0709. 2092] [INSPIRE].

[66] S. Hoche, F. Krauss, M. Schonherr and F. Siegert, Automating the POWHEG method in Sherpa, JHEP 04 (2011) 024 [arXiv: 1008.5399] [INSPIRE].

[67] K. Hamilton and P. Nason, Improving NLO-parton shower matched simulations with higher order matrix elements, JHEP 06 (2010) 039 [arXiv: 1004.1764] [INSPIRE].

[68] S. Hoche, F. Krauss, M. Schonherr and F. Siegert, NLO matrix elements and truncated showers, JHEP 08 (2011) 123 [arXiv: 1009.1127] [INSPIRE].

[69] N. Lavesson and L. Lönnblad, Extending CKKW-merging to one-loop matrix elements, JHEP 12 (2008) 070 [arXiv:0811.2912] [INSPIRE].

[70] S. Hoeche, F. Krauss, M. Schonherr and F. Siegert, QCD matrix elements + parton showers: The NLO case, arXiv:1207.5030 [INSPIRE].

[71] T. Gehrmann, S. Hoeche, F. Krauss, M. Schonherr and F. Siegert, NLO QCD matrix elements + parton showers in $e^{+} e^{-} \rightarrow$ hadrons, arXiv:1207.5031 [INSPIRE].

[72] ATLAS collaboration, Measurements of multijet production cross sections in proton-proton collisions at $7 \mathrm{TeV}$ center-of-mass energy with the ATLAS detector, ATLAS-CONF-2010-084 (2010).

[73] ATLAS collaboration, G. Aad et al., Measurement of the production cross section for $W$-bosons in association with jets in pp collisions at $\sqrt{s}=7 \mathrm{TeV}$ with the ATLAS detector, Phys. Lett. B 698 (2011) 325 [arXiv:1012.5382] [INSPIRE].

[74] ATLAS collaboration, S. Ask, Measurement of the production cross section for $W$ - and Z-bosons in association with jets in ATLAS, arXiv:1106.2061 [INSPIRE].

[75] ATLAS collaboration, G. Aad et al., Measurement of dijet production with a veto on additional central jet activity in pp collisions at $\sqrt{s}=7 \mathrm{TeV}$ using the ATLAS detector, JHEP 09 (2011) 053 [arXiv:1107.1641] [INSPIRE]. 
[76] ATLAS collaboration, G. Aad et al., Measurement of multi-jet cross sections in proton-proton collisions at a 7 TeV center-of-mass energy, Eur. Phys. J. C 71 (2011) 1763 [arXiv:1107.2092] [INSPIRE].

[77] CMS collaboration, S. Chatrchyan et al., Measurement of the Ratio of the 3-jet to 2-jet cross sections in pp collisions at $\sqrt{s}=7 \mathrm{TeV}$, Phys. Lett. B 702 (2011) 336 [arXiv:1106.0647] [INSPIRE].

[78] D0 collaboration, V.M. Abazov et al., Measurement of $Z / \gamma^{*}+$ jet $+X$ angular distributions in pp collisions at $\sqrt{s}=1.96 \mathrm{TeV}$, Phys. Lett. B 682 (2010) 370 [arXiv:0907.4286] [INSPIRE].

[79] CDF collaboration, T. Aaltonen et al., Measurement of inclusive jet cross-sections in $Z / \gamma^{*}\left(\rightarrow e^{+} e^{-}\right)+$jets production in p p collisions at $\sqrt{s}=1.96 \mathrm{TeV}$, Phys. Rev. Lett. 100 (2008) 102001 [arXiv:0711.3717] [INSPIRE].

[80] C. Berger et al., Next-to-leading order QCD predictions for $Z, \gamma^{*}+3$-jet distributions at the Tevatron, Phys. Rev. D 82 (2010) 074002 [arXiv:1004.1659] [INSPIRE].

[81] E. Kuraev and V.S. Fadin, Double logarithmical asymptotic of fermion inelastic form-factor in nonabelian gauge theory, Yad. Fiz. 27 (1978) 1107 [INSPIRE].

[82] D. Yennie, S.C. Frautschi and H. Suura, The infrared divergence phenomena and high-energy processes, Annals Phys. 13 (1961) 379 [INSPIRE].

[83] J.G.M. Gatheral, Exponentiation of eikonal cross-sections in nonabelian gauge theories, Phys. Lett. B 133 (1983) 90 [INSPIRE].

[84] J. Frenkel and J. Taylor, Nonabelian eikonal exponentiation, Nucl. Phys. B 246 (1984) 231 [INSPIRE].

[85] E. Laenen, L. Magnea and G. Stavenga, On next-to-eikonal corrections to threshold resummation for the Drell-Yan and DIS cross sections, Phys. Lett. B 669 (2008) 173 [arXiv:0807.4412] [INSPIRE].

[86] E. Laenen, G. Stavenga and C.D. White, Path integral approach to eikonal and next-to-eikonal exponentiation, JHEP 03 (2009) 054 [arXiv:0811.2067] [INSPIRE].

[87] O. Nachtmann and A. Reiter, A test for the gluon selfcoupling in the reactions $e^{+} e^{-} \rightarrow$ four jets and $Z^{0} \rightarrow$ four jets, $Z$. Phys. C 16 (1982) 45 [InSPIRE].

[88] M. Bengtsson and P. Zerwas, Four jet events in $e^{+} e^{-}$annihilation: testing the three gluon vertex, Phys. Lett. B 208 (1988) 306 [INSPIRE].

[89] S. Catani, Y.L. Dokshitzer, M. Olsson, G. Turnock and B. Webber, New clustering algorithm for multi-jet cross-sections in $e^{+} e^{-}$annihilation, Phys. Lett. B 269 (1991) 432 [INSPIRE].

[90] S. Schumann and F. Krauss, A parton shower algorithm based on Catani-Seymour dipole factorisation, JHEP 03 (2008) 038 [arXiv:0709.1027] [INSPIRE].

[91] A. Banfi, G. Corcella and M. Dasgupta, Angular ordering and parton showers for non-global QCD observables, JHEP 03 (2007) 050 [hep-ph/0612282] [INSPIRE].

[92] Y.L. Dokshitzer, V.A. Khoze, G. Marchesini and B. Webber, Measuring color flows in hard processes via hadronic correlations, Phys. Lett. B 245 (1990) 243 [INSPIRE].

[93] Y.L. Dokshitzer, G. Marchesini and G. Oriani, Measuring color flows in hard processes: beyond leading order, Nucl. Phys. B 387 (1992) 675 [INSPIRE]. 
[94] ATLAS collaboration, G. Aad et al., Measurement of the production cross section for $Z / \gamma^{*}$ in association with jets in pp collisions at $\sqrt{s}=7 \mathrm{TeV}$ with the ATLAS detector, Phys. Rev. D 85 (2012) 032009 [arXiv:1111.2690] [INSPIRE].

[95] S. Catani, B. Webber, Y.L. Dokshitzer and F. Fiorani, Average multiplicities in two and three jet $e^{+} e^{-}$annihilation events, Nucl. Phys. B 383 (1992) 419 [InSPIRE].

[96] R.M. Duran Delgado, J.R. Forshaw, S. Marzani and M.H. Seymour, The dijet cross section with a jet veto, JHEP 08 (2011) 157 [arXiv:1107.2084] [INSPIRE].

[97] T. Sjöstrand, A model for initial state parton showers, Phys. Lett. B 157 (1985) 321 [INSPIRE].

[98] M.H. Seymour, Matrix element corrections to parton shower algorithms, Comput. Phys. Commun. 90 (1995) 95 [hep-ph/9410414] [INSPIRE].

[99] V.S. Fadin, E. Kuraev and L. Lipatov, On the Pomeranchuk singularity in asymptotically free theories, Phys. Lett. B 60 (1975) 50 [InSPIRE].

[100] I. Balitsky and L. Lipatov, The Pomeranchuk singularity in quantum chromodynamics, Sov. J. Nucl. Phys. 28 (1978) 822 [Yad. Fiz. 28 (1978) 1597] [InSPIRE].

[101] M. Ciafaloni, Coherence effects in initial jets at small $Q^{2} / s$, Nucl. Phys. B 296 (1988) 49 [INSPIRE].

[102] S. Catani, F. Fiorani and G. Marchesini, Small x behavior of initial state radiation in perturbative QCD, Nucl. Phys. B 336 (1990) 18 [INSPIRE].

[103] B. Webber, Jet rates in deep inelastic scattering at small x, Phys. Lett. B 444 (1998) 81 [hep-ph/9810286] [INSPIRE].

[104] J.R. Forshaw and A. Sabio Vera, QCD coherence and jet rates in small $x$ deep inelastic scattering, Phys. Lett. B 440 (1998) 141 [hep-ph/9806394] [INSPIRE].

[105] G. Chachamis, M. Deak, A.S. Vera and P. Stephens, A comparative study of small x Monte Carlos with and without QCD coherence effects, Nucl. Phys. B 849 (2011) 28 [arXiv:1102.1890] [INSPIRE].

[106] S. Hoeche, F. Krauss, S. Schumann and F. Siegert, QCD matrix elements and truncated showers, JHEP 05 (2009) 053 [arXiv:0903.1219] [INSPIRE].

[107] S. Hoeche, S. Schumann and F. Siegert, Hard photon production and matrix-element parton-shower merging, Phys. Rev. D 81 (2010) 034026 [arXiv:0912.3501] [INSPIRE].

[108] M. Cacciari, G.P. Salam and G. Soyez, The anti-kt jet clustering algorithm, JHEP 04 (2008) 063 [arXiv: 0802.1189] [INSPIRE].

[109] SM AND NLO MULTILEG AND SM MC Working Groups collaboration,

J. Alcaraz Maestre et al., The SM and NLO multileg and SM MC working groups: summary report, arXiv:1203.6803 [INSPIRE].

[110] D0 collaboration, B. Abbott et al., Probing hard color-singlet exchange in p p $\bar{p}$ collisions at $\sqrt{s}=630 \mathrm{GeV}$ and $1800 \mathrm{GeV}$, Phys. Lett. B 440 (1998) 189 [hep-ex/9809016] [INSPIRE].

[111] ZEUS collaboration, M. Derrick et al., Rapidity gaps between jets in photoproduction at HERA, Phys. Lett. B 369 (1996) 55 [hep-ex/9510012] [INSPIRE].

[112] ZEUS collaboration, S. Chekanov et al., Photoproduction of events with rapidity gaps between jets at HERA, Eur. Phys. J. C 50 (2007) 283 [hep-ex/0612008] [INSPIRE]. 
[113] H1 collaboration, C. Adloff et al., Energy flow and rapidity gaps between jets in photoproduction at HERA, Eur. Phys. J. C 24 (2002) 517 [hep-ex/0203011] [INSPIRE].

[114] J.R. Andersen and J.M. Smillie, Constructing all-order corrections to multi-jet rates, JHEP 01 (2010) 039 [arXiv:0908.2786] [INSPIRE].

[115] J.R. Andersen and J.M. Smillie, Multiple jets at the LHC with high energy jets, JHEP 06 (2011) 010 [arXiv:1101.5394] [INSPIRE].

[116] S. Hoeche and M. Schonherr, Uncertainties in NLO + parton shower matched simulations of inclusive jet and dijet production, arXiv:1208.2815 [INSPIRE].

[117] U. Baur and E.N. Glover, Tagging the Higgs boson in $p p \rightarrow W^{+} W^{-} j j$, Phys. Lett. B 252 (1990) 683 [inSPIRE].

[118] V.D. Barger, K.-m. Cheung, T. Han and D. Zeppenfeld, Single forward jet tagging and central jet vetoing to identify the leptonic $W W$ decay mode of a heavy Higgs boson, Phys. Rev. D 44 (1991) 2701 [Erratum ibid. D 48 (1993) 5444] [INSPIRE].

[119] V.D. Barger, R. Phillips and D. Zeppenfeld, Mini-jet veto: a tool for the heavy Higgs search at the LHC, Phys. Lett. B 346 (1995) 106 [hep-ph/9412276] [INSPIRE].

[120] D.L. Rainwater, R. Szalapski and D. Zeppenfeld, Probing color singlet exchange in $Z+$ two jet events at the CERN LHC, Phys. Rev. D 54 (1996) 6680 [hep-ph/9605444] [INSPIRE].

[121] D.L. Rainwater, D. Summers and D. Zeppenfeld, Multi-jet structure of high $E_{T}$ hadronic collisions, Phys. Rev. D 55 (1997) 5681 [hep-ph/9612320] [INSPIRE].

[122] T. Figy, V. Hankele and D. Zeppenfeld, Next-to-leading order QCD corrections to Higgs plus three jet production in vector-boson fusion, JHEP 02 (2008) 076 [arXiv:0710.5621] [INSPIRE].

[123] B.E. Cox, J.R. Forshaw and A.D. Pilkington, Extracting Higgs boson couplings using a jet veto, Phys. Lett. B 696 (2011) 87 [arXiv:1006.0986] [INSPIRE].

[124] S. Catani, Y.L. Dokshitzer, F. Fiorani and B. Webber, Average number of jets in $e^{+} e^{-}$ annihilation, Nucl. Phys. B 377 (1992) 445 [INSPIRE].

[125] G. Leder, Jet fractions in $e^{+} e^{-}$annihilation, Nucl. Phys. B 497 (1997) 334 [hep-ph/9610552] [INSPIRE].

[126] B.R. Webber, QCD jets and parton showers, arXiv:1009.5871 [INSPIRE]. 